Article

\title{
Fourth-Order Comprehensive Adjoint Sensitivity Analysis Methodology for Nonlinear Systems (4th-CASAM-N): II. Application to a Nonlinear Heat Conduction Paradigm Model
}

Dan Gabriel Cacuci (D)

Citation: Cacuci, D.G. Fourth-Order Comprehensive Adjoint Sensitivity Analysis Methodology for Nonlinear Systems (4th-CASAM-N): II.

Application to a Nonlinear Heat Conduction Paradigm Model. J. Nucl. Eng. 2022, 3, 72-104. https:// doi.org/10.3390/jne3010005

Academic Editor: Brian Kiedrowski

Received: 7 January 2022

Accepted: 17 February 2022

Published: 24 February 2022

Publisher's Note: MDPI stays neutral with regard to jurisdictional claims in published maps and institutional affiliations.

Copyright: (C) 2022 by the author. Licensee MDPI, Basel, Switzerland. This article is an open access article distributed under the terms and conditions of the Creative Commons Attribution (CC BY) license (https:// creativecommons.org/licenses/by/ $4.0 /)$.
Center for Nuclear Science and Energy, University of South Carolina, Columbia, SC 29208, USA; cacuci@cec.sc.edu

\begin{abstract}
This work illustrates the application of the fourth-order comprehensive sensitivity analysis methodology for nonlinear systems (abbreviated as "4th-CASAM-N"), which enables the efficient computation of exactly determined 1st-, 2nd-, 3rd-, and 4th-order functional derivatives of results produced by computational models with respect to the model's parameters. Results produced by computational models are called model "responses" and the respective functional derivatives are called "sensitivities" (with respect) to model parameters. The qualifier "comprehensive" indicates that the 4th-CASAM-N methodology enables the exact and efficient computation not only of response sensitivities with respect to customary model parameters (including computational input data, correlations, initial and/or boundary conditions) but also with respect to imprecisely known material boundaries, as would be caused by manufacturing tolerances. The 4th-CASAM-N enables the hitherto very difficult, if not intractable, exact computation of all of the 1st-, 2nd-, 3rd-, and 4th-order response sensitivities for large-scale systems involving many parameters, as usually encountered in practice. A paradigm model that describes nonlinear heat conduction through a material has been chosen to illustrate the application of the 4th-CASAM-N methodology, as this model enables the derivation of tractable closed-form analytical expressions of representative 1st-, 2nd-, 3rd-, and 4th-order response sensitivities while largely avoiding side-tracking algebraic manipulations. The responses chosen for this paradigm model include not only physically measurable quantities but also a synthetic response designed to illustrate the enormous possible reduction in the number of computation when using the 4th-CASAM-N (rather than other methods) for computing response sensitivities.
\end{abstract}

Keywords: fourth-order adjoint sensitivity analysis methodology; efficient computation of exact sensitivities; first-order, second-order, third-order, fourth-order response sensitivities; nonlinear heat conduction

\section{Introduction}

The accompanying Part I [1] has presented the mathematical framework of the "fourthorder comprehensive sensitivity analysis methodology for nonlinear systems" (abbreviated as "4th-CASAM-N") methodology, which enables the most efficient computation of exactly determined expressions for (all of) the 1st-, 2nd-, 3rd-, and 4th-order response sensitivities. The 4th-CASAM-N is uniquely suited for the computation of sensitivities for large-scale systems involving many parameters: the larger the number of model parameters, the more efficient the 4th-CASAM-N. The principles underlying the application of the 4th-CASAM-N will be illustrated in this work by performing a fourth-order sensitivity analysis of a paradigm nonlinear heat conduction model, which enables the tractable computation of closed-form analytical expressions for representative sensitivities. A second-order adjoint sensitivity analysis of limited-scope has been performed on this illustrative model in [2,3] but the domain was considered to be perfectly well known, so that sensitivities to uncertain 
boundaries (such as would be caused by manufacturing tolerances) were not addressed, as the general theory for addressing such uncertain boundaries had not yet been developed (these theoretical capabilities were first developed in the accompanying Part I [1]). The system responses considered include the medium's temperature and thermal conductivity, which are physically measurable. In addition, this work also illustrates the enormous computational advantages that could be gained by taking advantage of particularities of the system/model under consideration, by considering a synthetically constructed model response, namely the square of the thermal conductivity, which is related to responses of interest (temperature, thermal conductivity) but which is not directly measurable. This synthetic response will be demonstrated to have only a finite number of non-zero sensitivities, as opposed to the temperature and thermal conductivity responses, which have infinitely many sensitivities. Therefore, computing the sensitivities of this synthetic response and subsequently obtaining the sensitivities of the responses of physical interest (namely the temperature and/or thermal conductivity) from the sensitivities of the synthetic response offers enormous computational advantages by comparison to computing directly the infinitely many sensitivities of the temperature and/or thermal conductivity.

This work is structured as follows: Section 2 presents the mathematical formulation of a simple nonlinear heat conduction model, which is characterized by uncertain (i.e., imprecisely known) model parameters and physical boundaries (as would arise from manufacturing tolerances). In Sections 3.1-3.4 of Section 3, the principles of applying the 4th-CASAM-N methodology are illustrated by presenting representative computations of 1st-, 2nd-, 3rd- and 4th-order response sensitivities. The discussion presented in Section 4 highlights the salient features of applying the 4th-CASAM-N methodology.

\section{Illustrative Model: Nonlinear Heat Conduction through a Slab}

To illustrate the main features of the 4th-CASAM-N, the illustrative model should represent an actual nonlinear physical system, yet be sufficiently simple so that the mathematical derivations should highlight (rather than obscure) the concepts underlying the 4th-CASAM-N. A model that fulfills these considerations is the following simple nonlinear model for the temperature distribution, $T(x)$, within a heated slab of homogeneous material of thickness $\ell$ :

$$
\begin{gathered}
\frac{d}{d x}\left[k(T) \frac{d T(x)}{d x}\right]+Q=0,0<x<\ell, \\
\frac{d T}{d x}=0, \text { at } x=0, \\
T(x)=0, \text { at } x=\ell .
\end{gathered}
$$

The thermal conductivity, $k(T)$, is considered to depend linearly on the temperature, having the following functional form:

$$
k(T)=k_{0}(1+\beta T),
$$

where $k_{0}\left[\mathrm{Wm}^{-1} \mathrm{C}^{-1}\right]=k(T=0)$ and $\beta\left[\mathrm{C}^{-1}\right]$ are experimentally measured quantities subject to uncertainties. As indicated by Equations (1)-(3), the slab is considered to be heated by a constant internal heat source of strength $Q\left[\mathrm{Wm}^{-3}\right]$. The left side of the slab, considered to be located at $x=0$, is considered to be insulated (for simplicity). The right side of the slab, considered to be located at $x=\ell$, is kept at a constant temperature, considered to be zero degrees, for simplicity.

A typical model response of interest would be the temperature inside the material, as measured by thermocouples placed at one (or several) location(s) denoted generically as $x_{r}$, $0<x_{r}<\ell$. Such a response is mathematically represented as follows:

$$
T_{r} \triangleq T\left(x_{r}\right)=\int_{0}^{\ell} T(x) \delta\left(x-x_{r}\right) d x, \quad 0<x_{r}<\ell,
$$


where $\delta\left(x-x_{r}\right)$ denotes the customary Dirac delta-functional. Another typical model response of interest is the slab's conductivity at a generic location $x_{r}, 0<x_{r}<\ell$. Such a response will be denoted as $k_{r} \triangleq k\left[T\left(x_{r}\right)\right]$ and is mathematically represented as follows:

$$
k_{r} \triangleq \int_{0}^{\ell} k[T(x)] \delta\left(x-x_{r}\right) d x=\int_{0}^{\ell} k_{0}(1+\beta T) \delta\left(x-x_{r}\right) d x, \quad 0<x_{r}<\ell .
$$

In summary, the imprecisely known parameters that characterize the heat conductivity model described in the foregoing are the quantities $Q, k_{0}, \beta$, the location $x_{r}$ of the response and the slab thickness $\ell$. These model parameters are considered to be components of the column vector $\boldsymbol{\alpha} \triangleq\left(\alpha_{1}, \alpha_{2}, \alpha_{3}, \alpha_{4}, \alpha_{5}\right)^{\dagger}$ defined as follows:

$$
\alpha \triangleq\left(\alpha_{1}, \alpha_{2}, \alpha_{3}, \alpha_{4}, \alpha_{5}\right)^{\dagger} \triangleq\left(Q, k_{0}, \beta, x_{r}, \ell\right)^{\dagger},
$$

with known nominal (or mean) values $\alpha^{0} \equiv\left(Q^{0}, k_{0}^{0}, \beta^{0}, x_{r}^{0}, \ell^{0}\right)^{\dagger}$. The dagger will be used throughout this work to denote "transposition." The model parameters, $\alpha$, are considered to be afflicted by uncertainties, so they can vary from their nominal values $\alpha^{0}$ by amounts represented by the components of the "vector of variations", $\delta \boldsymbol{\alpha} \triangleq \alpha-\boldsymbol{\alpha}^{0}$, which is defined as follows:

$$
\delta \boldsymbol{\alpha} \triangleq\left(\delta Q, \delta k_{0}, \delta \beta, \delta x_{r}, \delta \ell\right)^{\dagger}
$$

In practice, the variations $\delta Q, \delta k_{0}, \delta \beta, \delta x_{r}, \delta \ell$ are expressed in terms of the standard deviations that quantify the uncertainties in the respective model parameters. Such uncertainties arise because the mean values of $Q, q, k_{0}, \beta$ and $x_{r}$ are estimated based on measurements, while the geometrical dimension $\ell$ is subject to manufacturing tolerances.

The expression of the temperature distribution that satisfies Equations (1)-(3) can be obtained by using the Kirchoff transformation:

$$
U(x) \triangleq \int_{0}^{T} \frac{k\left(T^{\prime}\right)}{k_{0}} d T^{\prime}=T(x)+\frac{\beta}{2} T^{2}(x),
$$

which implies that

$$
\frac{d U}{d T}=\frac{k(T)}{k_{0}}, \frac{d T}{d x}=\frac{d T}{d U} \frac{d U}{d x}=\frac{k_{0}}{k(T)} \frac{d U}{d x} .
$$

Introducing the results obtained in Equation (10) into Equations (1)-(3) yields the following system of equations for $U(x)$ :

$$
\begin{gathered}
k_{0} \frac{d^{2} U}{d x^{2}}+Q=0,0<x<\ell, \\
\frac{d U(x)}{d x}=0, \text { at } x=0, \\
U(x)=0, \text { at } x=\ell .
\end{gathered}
$$

Solving the system of Equations (11)-(13) yields the following expression for $U(x)$ :

$$
U(x)=\frac{Q}{2 k_{0}}\left(\ell^{2}-x^{2}\right)>0 .
$$

It follows from the results obtained in Equations (9) and (14) that:

$$
T^{2}(x)=\frac{2}{\beta}[U(x)-T(x)] ; T(x)=\frac{1}{\beta}[-1+\sqrt{1+2 \beta U(x)}] .
$$


Inserting the result obtained in Equation (15) into Equation (5) yields the following closed-form expression for the response $T_{r}(\boldsymbol{\alpha})$ :

$$
T_{r}(\boldsymbol{\alpha})=\frac{1}{\beta}\left[-1+\sqrt{1+\frac{\beta Q}{k_{0}}\left(\ell^{2}-x_{r}^{2}\right)}\right] .
$$

Inserting the result obtained in Equation (15) into Equation (6) yields the following closed-form expression for the response $k_{r}(\boldsymbol{\alpha})$ :

$$
k_{r}(\boldsymbol{\alpha})=\left[k_{0}^{2}+k_{0} \beta Q\left(\ell^{2}-x_{r}^{2}\right)\right]^{1 / 2}
$$

At the nominal parameter values, the nominal values of the responses $T_{r}^{0} \triangleq T_{r}\left(\boldsymbol{\alpha}^{0}\right)$ and $k_{r}^{0} \triangleq k_{r}\left(\boldsymbol{\alpha}^{0}\right)$, respectively, are denoted as follows:

$$
T_{r}^{0} \triangleq T_{r}\left(\alpha^{0}\right)=\left\{\frac{1}{\beta}\left[-1+\sqrt{1+\frac{\beta Q}{k_{0}}\left(\ell^{2}-x_{r}^{2}\right)}\right]\right\}_{\alpha^{0}},
$$

and

$$
k_{r}^{0} \triangleq k_{r}\left(\boldsymbol{\alpha}^{0}\right)=\left\{\left[k_{0}^{2}+k_{0} \beta Q\left(\ell^{2}-x_{r}^{2}\right)\right]^{1 / 2}\right\}_{\boldsymbol{\alpha}^{0}} .
$$

The notation \{\}$_{\alpha^{0}}$, which appears in Equations (18) and (19), will be used in this work to indicate that the quantity within the respective braces is to be evaluated at the nominal values of the parameters, and implicitly, at the nominal value of the respective state function(s).

The closed-form analytical expression for the state function $T(x)$ provided in Equation (15) is not available in practice. Consequently, the closed-form analytical expressions for response $k_{r}$ provided in Equation (17) are not available in practice, either. In practice, the model's state functions and responses need to be computed numerically.

\section{Illustrative Application of the 4th-CASAM-N to the Nonlinear Conduction Model}

The concepts underlying the 4th-CASAM-N will be illustrated in this section by considering the nonlinear heat conduction model presented in Section 2, above. The derivation of the exact expressions of the first-order sensitivities of the temperature response with respect to the model's parameters will be presented in Sections 3.1-3.4 illustrate the derivation of the exact expressions of representative 2 nd-, 3rd-, and 4 th-order sensitivities of the temperature response with respect to selected model parameters.

\subsection{First-Order Response Sensitivities}

The 1st-order sensitivities of the temperature and thermal conductivity response defined in Equations (5) and (6), respectively, will be derived in this section by applying the principles of the 4th-CASAM-N methodology. Both of these responses are linear functions of the state function (temperature). In addition, therefore, this section will also present the illustrative application of the 4th-CASAM-N methodology to derive the expressions of the 1st-order sensitivities of an illustrative model response, which is a nonlinear function of the state function (temperature).

\subsubsection{First-Order Sensitivities of the Forward State Function (Temperature)}

The first-order sensitivities of the response $T_{r}(\boldsymbol{\alpha})$ with respect to parameter variations $\delta \boldsymbol{\alpha}$, are obtained by determining the first-order Gateaux- (G-)differential $\delta T_{r}\left(T^{0} ; \boldsymbol{\alpha}^{0} ; \delta T ; \delta \boldsymbol{\alpha}\right)$ 
of $T_{r}(\boldsymbol{\alpha})$ at the nominal parameter values. Thus, applying the definition of the G-differential to Equation (5) yields the following relation:

$$
\begin{aligned}
& \delta T_{r}\left(T^{0} ; \boldsymbol{\alpha}^{0} ; \delta T ; \delta \boldsymbol{\alpha}\right) \triangleq\left[\frac{d}{d \varepsilon} \int_{0}^{\ell^{0}+\varepsilon \delta \ell}\left[T^{0}(x)+\varepsilon \delta T(x)\right] \delta\left[x-\left(x_{r}^{0}+\varepsilon \delta x_{r}\right)\right] d x\right]_{\varepsilon=0} \\
& \triangleq\left\{\delta T_{r}\left(\boldsymbol{\alpha}^{0} ; \delta \boldsymbol{\alpha}\right)\right\}_{d i r}+\left\{\delta T_{r}\left(\boldsymbol{\alpha}^{0} ; \delta T\right)\right\}_{i n d^{\prime}}
\end{aligned}
$$

where the direct-effect term $\left\{\delta T_{r}\left(T^{0} ; \boldsymbol{\alpha}^{0} ; \delta \boldsymbol{\alpha}\right)\right\}_{\text {dir }}$ depends only on parameter variations and is defined as follows:

$$
\left\{\delta T_{r}\left(T^{0} ; \boldsymbol{\alpha}^{0} ; \delta \boldsymbol{\alpha}\right)\right\}_{d i r} \triangleq-\left(\delta x_{r}\right)\left\{\int_{0}^{\ell} T(x) \delta^{\prime}\left(x-x_{r}\right) d x\right\}_{\boldsymbol{\alpha}^{0}}
$$

and where the indirect-effect term $\left\{\delta T_{r}\left(\boldsymbol{\alpha}^{0} ; \delta T\right)\right\}_{\text {ind }}$ depends only on variations $\delta T(x)$ in the state function $T(x)$ and is defined as follows:

$$
\left\{\delta T_{r}\left(\boldsymbol{\alpha}^{0} ; \delta T\right)\right\}_{\text {ind }} \triangleq\left\{\int_{0}^{\ell} \delta T(x) \delta\left(x-x_{r}\right) d x\right\}_{\boldsymbol{\alpha}^{0}} .
$$

The direct-effect term defined by Equation (21) can be evaluated immediately by using the expression provided in Equation (15) for the state function $T(x)$. In practice, the direct-effect term defined by Equation (21) is computed numerically, by using the numerically computed values for $T(x)$. On the other hand, the indirect-effect term defined by Equation (22) can be evaluated only after having determined the function $\delta T(x)$, which is the solution of the G-differentiated model comprising Equations (1)-(3). Applying the definition of the G-differential to Equations (1)-(3) yields the following system:

$$
\begin{gathered}
\left\{\frac{d}{d \varepsilon} \frac{d}{d x}\left[\left(k_{0}^{0}+\varepsilon \delta k_{0}\right)+\left(k_{0}^{0}+\varepsilon \delta k_{0}\right)\left(\beta^{0}+\varepsilon \delta \beta\right)\left(T^{0}+\varepsilon \delta T\right)\right] \frac{d\left(T^{0}+\varepsilon \delta T\right)}{d x}\right\}_{\varepsilon=0} \\
+\left\{\frac{d}{d \varepsilon}\left(Q^{0}+\varepsilon \delta Q\right)\right\}_{\varepsilon=0}=0,0<x<\ell^{0} ; \\
\left\{\frac{d}{d \varepsilon} \frac{d\left(T^{0}+\varepsilon \delta T\right)}{d x}\right\}_{\varepsilon=0}=0, \text { at } x=0 ; \\
\left\{\frac{d}{d \varepsilon}\left[T^{0}\left(\ell^{0}+\varepsilon \delta \ell\right)+\varepsilon \delta T\right]\right\}_{\varepsilon=0}=0, \text { at } x=\ell^{0} .
\end{gathered}
$$

Performing the differentiations with respect to $\varepsilon$ in Equations (23)-(25) and setting $\varepsilon=0$ in the resulting expressions yields the following system of equations:

$$
\begin{gathered}
\left\{\frac{d^{2}}{d x^{2}}[k(T) \delta T(x)]\right\}_{\boldsymbol{\alpha}^{0}}=\left\{s_{V}^{(1)}(T ; \boldsymbol{\alpha} ; \delta \boldsymbol{\alpha})\right\}_{\boldsymbol{\alpha}^{0^{0}}}, 0<x<\ell^{0} \\
\frac{d[\delta T(x)]}{d x}=0, \text { at } x=0 ; \\
\delta T(x)+(\delta \ell)\left\{\frac{d T(x)}{d x}\right\}_{\boldsymbol{\alpha}^{0}}=0, \text { at } x=\ell^{0}
\end{gathered}
$$

where:

$$
s_{V}^{(1)}(T ; \boldsymbol{\alpha} ; \delta \boldsymbol{\alpha}) \triangleq-(\delta Q)+\left(\delta k_{0}\right) \frac{Q}{k_{0}}-(\delta \beta) k_{0} \frac{d}{d x}\left[T(x) \frac{d T(x)}{d x}\right] .
$$

The system of equations shown in Equations (26)-(28) will be called the first-level Variational Sensitivity System (1st-LVSS). Rather than solving the 1st-LVSS repeatedly, to obtain 
the function $\delta T(x)$ for all parameter variations, it is more advantageous computationally to derive an alternative expression for the indirect-effect term defined by Equation (22), which does not depend on $\delta T(x)$. This alternative expression for the indirect-effect term will be obtained by using the first-level adjoint sensitivity system (1st-LASS), which is adjoint to the 1st-LVSS defined by Equations (26)-(28). The Hilbert space appropriate for constructing the 1st-LASS will be denoted as $H_{1}(\Omega) \equiv L_{2}(\Omega)$ and comprises all square integrable (in the sense of Lebesgue) functions $a(x)$ defined on the domain $x \in \Omega \equiv[0, \ell]$ and endowed with the following inner product for two functions $a_{1}(x) \in H_{1}(\Omega)$ and $a_{2}(x) \in H_{1}(\Omega)$ :

$$
\left\langle a_{1}(x), a_{2}(x)\right\rangle_{1} \triangleq\left\{\int_{0}^{\ell} a_{1}(x) a_{2}(x) d x\right\}_{\alpha^{0}}
$$

Presently forming the inner product of Equation (26) with a yet undefined function $\psi^{(1)}(x) \in H_{1}(\Omega)$ and integrating the resulting equations twice by parts to transfer the differential operations from $\delta T(x)$ to $\psi^{(1)}(x)$ yields the following relation:

$$
\begin{aligned}
& \left\{\int_{0}^{\ell} \psi^{(1)}(x) \frac{d^{2}}{d x^{2}}[k(T) \delta T(x)] d x\right\}_{\boldsymbol{\alpha}^{0}}=\left\{\int_{0}^{\ell} \psi^{(1)}(x) s_{V}^{(1)}(T ; \boldsymbol{\alpha} ; \delta \boldsymbol{\alpha}) d x\right\}_{\boldsymbol{\alpha}^{0}} \\
& =\left\{\psi^{(1)}(x) \frac{d}{d x}\left[k\left(T^{0}\right) \delta T(x)\right]-\delta T(x) k\left(T^{0}\right) \frac{d \psi^{(1)}(x)}{d x}\right\}_{x=0}^{x=\ell} \\
& +\left\{\int_{0}^{\ell} \delta T(x)\left[k(T) \frac{d^{2} \psi^{(1)}(x)}{d x^{2}}\right] d x\right\}_{\boldsymbol{\alpha}^{0}} .
\end{aligned}
$$

The last term on the right-side of Equation (31) will represent the indirect-effect term defined in Equation (22) and the unknown values of $\delta T(x)$ will be eliminated from Equation (31) by defining the following first-level adjoint sensitivity system (1st-LASS) for the 1st-level adjoint function $a^{(1)}(x)$ :

$$
\begin{gathered}
\left\{k(T) \frac{d^{2} \psi^{(1)}(x)}{d x^{2}}\right\}_{\alpha^{0}}=\left\{\delta\left(x-x_{r}\right)\right\}_{\alpha^{0}}, 0<x<\ell^{0}, \\
\psi^{(1)}(x)=0, \text { at } x=\ell^{0} \\
\frac{d \psi^{(1)}(x)}{d x}=0, \text { at } x=0 .
\end{gathered}
$$

Using the relations that constitute the 1st-LVSS and the 1st-LASS together with Equation (22) in Equation (31) transforms the latter relation into the following form:

$$
\left\{\delta T_{r}\left(T^{0} ; \boldsymbol{\alpha}^{0} ; \delta T\right)\right\}_{\text {ind }}=\left\{\int_{0}^{\ell} \psi^{(1)}(x) s_{V}^{(1)}(T ; \boldsymbol{\alpha} ; \delta \boldsymbol{\alpha}) d x\right\}_{\boldsymbol{\alpha}^{0}}-(\delta \ell)\left\{\left[k(T) \frac{d T(x)}{d x} \frac{d \psi^{(1)}(x)}{d x}\right]_{x=\ell}\right\}_{\boldsymbol{\alpha}^{0}} .
$$

Adding the expressions obtained in Equations (21) and (35) yields the following expression for the total 1st-order G-differential $\delta T_{r}\left(T^{0} ; \boldsymbol{\alpha}^{0} ; \delta T ; \delta \boldsymbol{\alpha}\right)$ :

$$
\begin{aligned}
& \delta T_{r}\left(T^{0} ; \boldsymbol{\alpha}^{0} ; \delta T ; \delta \boldsymbol{\alpha}\right)=-\left(\delta x_{r}\right)\left\{\int_{0}^{\ell} T(x) \delta^{\prime}\left(x-x_{r}\right) d x\right\}_{\boldsymbol{\alpha}^{0}}+\left\{\int_{0}^{\ell} \psi^{(1)}(x) s_{V}^{(1)}(T ; \boldsymbol{\alpha} ; \delta \boldsymbol{\alpha}) d x\right\}_{\boldsymbol{\alpha}^{0}} \\
& -(\delta \ell)\left\{\left[k(T) \frac{d T(x)}{d x} \frac{d a^{(1)}(x)}{d x}\right]_{x=\ell}\right\}_{\boldsymbol{\alpha}^{0}} \triangleq \frac{\partial T_{r}}{\partial Q}(\delta Q)+\frac{\partial T_{r}}{\partial k_{0}}\left(\delta k_{0}\right)+\frac{\partial T_{r}}{\partial \beta}(\delta \beta)+\frac{\partial T_{r}}{\partial x_{r}}\left(\delta x_{r}\right)+\frac{\partial T_{r}}{\partial \ell}(\delta \ell) .
\end{aligned}
$$

Replacing in Equation (36) the expression for $s_{V}^{(1)}(T ; \boldsymbol{\alpha} ; \delta \boldsymbol{\alpha})$ from Equation (29) and identifying the quantities that multiply the respective parameter variations yields the following 
expressions for the 1st-order sensitivities of the temperature response with respect to the model parameters:

$$
\begin{aligned}
& \frac{\partial T_{r}}{\partial Q}=-\left\{\int_{0}^{\ell} \psi^{(1)}(x) d x\right\}_{\alpha^{0}} \\
& \frac{\partial T_{r}}{\partial k_{0}}=\left\{\frac{Q}{k_{0}} \int_{0}^{\ell} \psi^{(1)}(x) d x\right\}_{\alpha^{0}}, \\
& \frac{\partial T_{r}}{\partial \beta}=-\left\{k_{0} \int_{0}^{\ell} \psi^{(1)}(x) \frac{d}{d x}\left[T(x) \frac{d T(x)}{d x}\right] d x\right\}_{\alpha^{0}}, \\
& \frac{\partial T_{r}}{\partial x_{r}}=-\left\{\int_{0}^{\ell} T(x) \delta^{\prime}\left(x-x_{r}\right) d x\right\}_{\alpha^{0}}=\left\{\int_{0}^{\ell} \frac{T(x)}{d x} \delta\left(x-x_{r}\right) d x\right\}_{\alpha^{0}}, \\
& \frac{\partial T_{r}}{\partial \ell}=-\left\{\left[k(T) \frac{d T(x)}{d x} \frac{d \psi^{(1)}(x)}{d x}\right]_{x=\ell}\right\}_{\alpha^{0}} .
\end{aligned}
$$

After solving the 1st-LASS to determine the 1st-level adjoint function $\psi^{(1)}(x)$, the expressions obtained in Equations (37)-(41) indicate that all of the 1st-order response sensitivities can be computed exactly and efficiently, using just quadrature (integrations) formulas.

It is important to note that the 1st-LASS is independent of parameter variations. Solving the 1st-LASS yields the following 1st- level adjoint function $\psi^{(1)}(x)$ :

$$
\psi^{(1)}(x)=\left\{\frac{1}{k_{r}(\boldsymbol{\alpha})}\left[\left(x-x_{r}\right) H\left(x-x_{r}\right)-\ell+x_{r}\right]\right\}_{\boldsymbol{\alpha}^{0}} .
$$

where $H(x)$ is the customary Heaviside unit-step functional, defined as $H(x)=0$ if $x<0$; $H(x)=1$ if $x \geq 0$. Using the expressions obtained in Equations (16) and (42) in Equations (37)-(41) and carrying out the respective integrations yields the following detailed, exact expressions for the 1st-order sensitivities of the temperature response $T_{r}(\boldsymbol{\alpha})$ with respect to the model parameters:

$$
\begin{gathered}
\frac{\partial T_{r}(\boldsymbol{\alpha})}{\partial Q}=\frac{\ell^{2}-x_{r}^{2}}{2 k_{r}(\boldsymbol{\alpha})}, \\
\frac{\partial T_{r}(\boldsymbol{\alpha})}{\partial k_{0}}=\frac{Q}{k_{0}} \frac{x_{r}^{2}-\ell^{2}}{2 k_{r}(\boldsymbol{\alpha})}, \\
\frac{\partial T_{r}(\boldsymbol{\alpha})}{\partial \beta}=\frac{1}{\beta^{2}}-\frac{1}{\beta} \frac{1}{2 k_{r}(\boldsymbol{\alpha})}\left[2 \frac{k_{0}}{\beta}+Q\left(\ell^{2}-x_{r}^{2}\right)\right], \\
\frac{\partial T_{r}(\boldsymbol{\alpha})}{\partial x_{r}}=\frac{-Q x_{r}}{k_{r}(\boldsymbol{\alpha})}, \\
\frac{\partial T_{r}(\boldsymbol{\alpha})}{\partial \ell}=\frac{Q \ell}{k_{r}(\boldsymbol{\alpha})} .
\end{gathered}
$$

All of the expressions of the 1st-order sensitivities presented in Equations (43)-(47) are to be evaluated at the respective nominal parameter values but the indicator \{\}$_{\alpha^{0}}$ has been omitted, for simplicity.

\subsubsection{First-Order Sensitivities of a Linear Response (Thermal Conductivity)}

In addition to the temperature, another important response is the thermal conductivity of the material in which heat is being transported, i.e., the function $k_{r}(\boldsymbol{\alpha})$ at a generic location $x_{r}, 0<x_{r}<\ell$. In this illustrative model, the thermal conductivity is a linear 
function of the temperature, so the sensitivity analysis of the thermal conductivity serves to illustrate the application of the 4th-CASAM-N to a linear model response. The first-order sensitivities of the response $k_{r}(\boldsymbol{\alpha})$ with respect to parameter variations $\delta \alpha$ are obtained by determining the first-order Gateaux- (G-) differential $\delta k_{r}\left(T^{0} ; \boldsymbol{\alpha}^{0} ; \delta T ; \delta \boldsymbol{\alpha}\right)$ of $k_{r}(\boldsymbol{\alpha})$ at the nominal parameter values. Thus, applying the definition of the G-differential to Equation (6) yields the following relation:

$$
\begin{aligned}
& \delta k_{r}\left(T^{0} ; \boldsymbol{\alpha}^{0} ; \delta T ; \delta \boldsymbol{\alpha}\right) \triangleq\left\{\frac{d}{d \varepsilon} \int_{0}^{\ell^{0}+\varepsilon \delta \ell}\left(k_{0}^{0}+\varepsilon \delta k_{0}\right)\left[1+\left(\beta^{0}+\varepsilon \delta \beta\right)\left(T^{0}+\varepsilon \delta T\right)\right]\right. \\
& \left.\times \delta\left[x-\left(x_{r}^{0}+\varepsilon \delta x_{r}\right)\right] d x\right\}_{\varepsilon=0} \triangleq\left\{\delta k_{r}\left(T^{0} ; \boldsymbol{\alpha}^{0} ; \delta \boldsymbol{\alpha}\right)\right\}_{\text {dir }}+\left\{\delta k_{r}\left(T^{0} ; \boldsymbol{\alpha}^{0} ; \delta T\right)\right\}_{\text {ind }}
\end{aligned}
$$

where the direct-effect term $\left\{\delta k_{r}\left(T^{0} ; \boldsymbol{\alpha}^{0} ; \delta \boldsymbol{\alpha}\right)\right\}_{\text {dir }}$ depends only on parameter variations and is defined as follows:

$$
\begin{aligned}
& \left\{\delta k_{r}\left(T^{0} ; \boldsymbol{\alpha}^{0} ; \delta \boldsymbol{\alpha}\right)\right\}_{d i r} \triangleq\left(\delta k_{0}\right)\left\{\int_{0}^{\ell}[1+\beta T(x)] \delta\left(x-x_{r}\right) d x\right\}_{\boldsymbol{\alpha}^{0}} \\
& +(\delta \beta)\left\{k_{0} \int_{0}^{\ell} T(x) \delta\left(x-x_{r}\right) d x\right\}_{\boldsymbol{\alpha}^{0}}-\left(\delta x_{r}\right)\left\{k_{0} \int_{0}^{\ell}[1+\beta T(x)] \delta^{\prime}\left(x-x_{r}\right) d x\right\}_{\boldsymbol{\alpha}^{0}},
\end{aligned}
$$

and where the indirect-effect term $\left\{\delta k_{r}\left(T^{0} ; \boldsymbol{\alpha}^{0} ; \delta T\right)\right\}_{\text {ind }}$ depends only on variations $\delta T(x)$ in the state function $T(x)$ and is defined as follows:

$$
\left\{\delta k_{r}\left(T^{0} ; \alpha^{0} ; \delta T\right)\right\}_{\text {ind }} \triangleq\left\{k_{0} \beta \int_{0}^{\ell} \delta T(x) \delta\left(x-x_{r}\right) d x\right\}_{\boldsymbol{\alpha}^{0}} .
$$

The direct-effect term defined by Equation (49) can be evaluated immediately by replacing the expression provided in Equation (15) for the state function $T(x)$. In practice, the direct-effect term defined by Equation (49) is computed numerically, by using the numerically computed values for $T(x)$. The indirect-effect term defined by Equation (50) can be evaluated only after having determined the function $\delta T(x)$, which is the solution of the 1st-LVSS represented by Equations (26)-(28). However, the indirect-effect term is obtainable by simply multiplying the expression obtained in Equation (35) by the product of parameters $k_{0} \beta$. As all of the operations for obtaining the 1st-order sensitivities of $k_{r}(\boldsymbol{\alpha})$ are essentially duplications of the mathematical operations performed in Section 3.1.1, the respective details will be omitted; only the expressions of the 1st-order sensitivities of $k_{r}(\boldsymbol{\alpha})$ are presented below:

$$
\begin{gathered}
\frac{\partial k_{r}}{\partial Q}=-\left\{\int_{0}^{\ell} \varphi^{(1)}(x) d x\right\}_{\alpha^{0}} \\
\frac{\partial k_{r}}{\partial k_{0}}=\left\{\int_{0}^{\ell}[1+\beta T(x)] \delta\left(x-x_{r}\right) d x\right\}_{\alpha^{0}}^{\ell}+\left\{\frac{Q}{k_{0}} \int_{0}^{\ell} \varphi^{(1)}(x) d x\right\}_{\alpha^{0}} \\
\frac{\partial k_{r}}{\partial \beta}=\left\{\begin{array}{c}
\ell \\
\left.k_{0} \int_{0}^{\ell} T(x) \delta\left(x-x_{r}\right) d x\right\}_{\alpha^{0}}-k_{0} \varphi^{(1)}(x) \frac{d}{d x}\left[T(x) \frac{d T(x)}{d x}\right] d x \\
\frac{\partial k_{r}}{\partial x_{r}}=-\left\{k_{0}[1+\beta T(x)] \delta^{\prime}\left(x-x_{r}\right) d x\right. \\
\frac{\partial k_{r}}{\partial \ell}=-\left\{\left[k(T) \frac{d T(x)}{d x} \frac{d \varphi^{(1)}(x)}{d x}\right\}_{x=\ell}\right\}_{\alpha^{0}}
\end{array}\right.
\end{gathered}
$$


The 1st-level adjoint function $\varphi^{(1)}(x)$, which appears in the expressions presented in Equations (51)-(55) is the solution of the following first-level adjoint sensitivity system (1st-LASS):

$$
\begin{gathered}
\left\{k(T) \frac{d^{2} \varphi^{(1)}(x)}{d x^{2}}\right\}_{\alpha^{0}}=\left\{k_{0} \beta \delta\left(x-x_{r}\right)\right\}_{\alpha^{0}}, 0<x<\ell^{0} \\
\varphi^{(1)}(x)=0, \text { at } x=\ell^{0} \\
\frac{d \varphi^{(1)}(x)}{d x}=0, \text { at } x=0 .
\end{gathered}
$$

The explicit expression of the 1st-level adjoint function $\varphi^{(1)}(x)$, obtained by solving Equations (56)-(58), is as follows:

$$
\varphi^{(1)}(x)=\left\{\frac{k_{0} \beta}{k\left[T\left(x_{r}\right)\right]}\left[\left(x-x_{r}\right) H\left(x-x_{r}\right)-\ell+x_{r}\right]\right\}_{\alpha^{0}} .
$$

Introducing in Equations (51)-(55) the right side of Equation (59) and performing the various integrations yields the closed-form expressions for the 1st-order sensitivities of $k_{r}(\boldsymbol{\alpha})$.

If the results for the 1st-order sensitivities of $T_{r}(\boldsymbol{\alpha})$, i.e., the results produced by numerically computing the right sides of Equations (37)-(41) or Equations (43)-(47), are available, then the sensitivities of $k_{r}(\boldsymbol{\alpha})$ can be computed directly from the sensitivities of $T_{r}(\boldsymbol{\alpha})$ and vice versa, by using the G-differential of Equation (4), which reads as follows:

$$
\delta k(T)=\delta k_{0}(1+\beta T)+(\delta \beta) k_{0} T+k_{0} \beta(\delta T) .
$$

In particular, the relation provided in Equation (60) indicates that the sensitivities of $k_{r}(\boldsymbol{\alpha})$ are related to the sensitivities of $T_{r}(\boldsymbol{\alpha})$ as follows:

$$
\begin{gathered}
\frac{\partial k_{r}(\boldsymbol{\alpha})}{\partial Q}=k_{0} \beta \frac{\partial T_{r}(\boldsymbol{\alpha})}{\partial Q}=k_{0} \beta \frac{\ell^{2}-x_{r}^{2}}{2 k_{r}(\boldsymbol{\alpha})}, \\
\frac{\partial k_{r}(\boldsymbol{\alpha})}{\partial k_{0}}=\left[1+\beta T_{r}(\boldsymbol{\alpha})\right]+k_{0} \beta \frac{\partial T_{r}(\boldsymbol{\alpha})}{\partial k_{0}}=\frac{k_{0}}{k_{r}(\boldsymbol{\alpha})}+\beta Q \frac{\ell^{2}-x_{r}^{2}}{2 k_{r}(\boldsymbol{\alpha})}, \\
\frac{\partial k_{r}(\boldsymbol{\alpha})}{\partial \beta}=k_{0} T_{r}(\boldsymbol{\alpha})+k_{0} \beta \frac{\partial T_{r}(\boldsymbol{\alpha})}{\partial \beta}=\frac{k_{0} Q\left(\ell^{2}-x_{r}^{2}\right)}{2 k_{r}(\boldsymbol{\alpha})}, \\
\frac{\partial k_{r}(\boldsymbol{\alpha})}{\partial x_{r}}=k_{0} \beta \frac{\partial T_{r}(\boldsymbol{\alpha})}{\partial x_{r}}=-\frac{k_{0} \beta Q x_{r}}{k_{r}(\boldsymbol{\alpha})}, \\
\frac{\partial k_{r}}{\partial \ell}=k_{0} \beta \frac{\partial T_{r}}{\partial \ell}=\frac{k_{0} \beta Q \ell}{k_{r}(\boldsymbol{\alpha})} .
\end{gathered}
$$

All of the expressions of the 1st-order sensitivities presented in Equations (61)-(65) are to be evaluated at the respective nominal parameter values but the indicator \{\}$_{\alpha^{0}}$ has been omitted, for simplicity.

\subsubsection{First-Order Sensitivities of a Nonlinear Response}

A paradigm example of a nonlinear response is the square of the thermal conductivity, namely $k_{r}^{2}(\boldsymbol{\alpha})$. The remainder of this subsection will present the sensitivity analysis of this response, which will be denoted as $R(T, \boldsymbol{\alpha}) \triangleq k_{r}^{2}(\boldsymbol{\alpha})$, because it also demonstrates (as will be shown in the remainder of this subsection) that particularly chosen responses offer significant computational advantages over performing the direct sensitivity analysis of the 
response of actual interest, which would be $k_{r}(\boldsymbol{\alpha})$. The expression defining the response $R(T, \boldsymbol{\alpha}) \triangleq k_{r}^{2}(\boldsymbol{\alpha})$ is as follows:

$$
R(T, \boldsymbol{\alpha})=\int_{0}^{\ell}\left[k_{0}(1+\beta T)\right]^{2} \delta\left(x-x_{r}\right) d x=k_{0}^{2}+k_{0} \beta Q\left(\ell^{2}-x_{r}^{2}\right), \quad 0<x_{r}<\ell .
$$

It is evident from the closed-form expression provided in Equation (66) that $R(T, \boldsymbol{\alpha}) \triangleq$ $k_{r}^{2}(\boldsymbol{\alpha})$ is a polynomial of fifth-order in the model parameters, so all of the sensitivities of order higher than five will vanish identically. In contradistinction to the vanishing of the sensitivities of order higher than five for the response $R(T, \boldsymbol{\alpha}) \triangleq k_{r}^{2}(\boldsymbol{\alpha})$, the sensitivities of the temperature response, $T_{r}(\boldsymbol{\alpha})$, and-consequently-of the thermal conductivity response, $k_{r}(\boldsymbol{\alpha})$, do not vanish identically, but exist for all orders, because of the fractional power (square root) involved in their expressions, cf. Equations (16) and (17). For the 1st-order sensitivities, a single adjoint computation is required per response to obtain the 1st-level adjoint function and subsequently compute efficiently and exactly all of the response's 1st-order sensitivities, regardless of the number of model parameters, so if only the 1st-order sensitivities of $T_{r}(\boldsymbol{\alpha})$ and/or $k_{r}(\boldsymbol{\alpha})$, they would be most advantageously computed by using the respective 1st-LASS. If, however, the high-order sensitivities of $T_{r}(\boldsymbol{\alpha})$ and/or $k_{r}(\alpha)$ are also of interest, then it is considerably more advantageous to compute the higher-order sensitivities of $R(T, \boldsymbol{\alpha}) \triangleq k_{r}^{2}(\boldsymbol{\alpha})$, by applying the 4th-CASAM-N, and subsequently determine the high-order sensitivities of $T_{r}(\boldsymbol{\alpha})$ and /or $k_{r}(\boldsymbol{\alpha})$ through their relations to the sensitivities of $R(T, \boldsymbol{\alpha}) \triangleq k_{r}^{2}(\boldsymbol{\alpha})$, which are as follows:

(i) First-order variations:

$$
\delta R(T, \boldsymbol{\alpha})=2 k_{r}(\boldsymbol{\alpha}) \delta k_{r}(\boldsymbol{\alpha}) ; \Rightarrow \delta k_{r}(\boldsymbol{\alpha})=\delta R(T, \boldsymbol{\alpha}) / 2 k_{r}(\boldsymbol{\alpha}) ;
$$

(ii) Second-order variations:

$$
\begin{gathered}
\delta^{2} R(T, \boldsymbol{\alpha})=2\left[\delta k_{r}(\boldsymbol{\alpha})\right]^{2}+2 k_{r}(\boldsymbol{\alpha}) \delta^{2} k_{r}(\boldsymbol{\alpha}) ; \\
\Rightarrow \delta^{2} k_{r}(\boldsymbol{\alpha})=\frac{\delta^{2} R(T, \boldsymbol{\alpha})-2\left[\delta k_{r}(\boldsymbol{\alpha})\right]^{2}}{2 k_{r}(\boldsymbol{\alpha})}
\end{gathered}
$$

(iii) Third-order variations:

$$
\begin{aligned}
& \delta^{3} R(T, \boldsymbol{\alpha})=4 \delta k_{r}(\boldsymbol{\alpha}) \delta^{2} k_{r}(\boldsymbol{\alpha})+2 \delta k_{r}(\boldsymbol{\alpha}) \delta^{2} k_{r}(\boldsymbol{\alpha})+2 k_{r}(\boldsymbol{\alpha}) \delta^{3} k_{r}(\boldsymbol{\alpha}) \\
& \Rightarrow \delta^{3} k_{r}(\boldsymbol{\alpha})=\frac{\delta^{3} R(T, \boldsymbol{\alpha})-4 \delta k_{r}(\boldsymbol{\alpha}) \delta^{2} k_{r}(\boldsymbol{\alpha})-2 \delta k_{r}(\boldsymbol{\alpha}) \delta^{2} k_{r}(\boldsymbol{\alpha})}{2 k_{r}(\boldsymbol{\alpha})}
\end{aligned}
$$

(iv) Fourth-order variations:

$$
\begin{aligned}
& \delta^{4} R(T, \boldsymbol{\alpha})=6\left[\delta^{2} k_{r}(\boldsymbol{\alpha})\right]^{2}+8 \delta k_{r}(\boldsymbol{\alpha}) \delta^{3} k_{r}(\boldsymbol{\alpha})+2 k_{r}(\boldsymbol{\alpha}) \delta^{4} k_{r}(\boldsymbol{\alpha}) \\
& \Rightarrow \delta^{4} k_{r}(\boldsymbol{\alpha})=\frac{\delta^{4} R(T, \boldsymbol{\alpha})-6\left[\delta^{2} k_{r}(\boldsymbol{\alpha})\right]^{2}-8 \delta k_{r}(\boldsymbol{\alpha}) \delta^{3} k_{r}(\boldsymbol{\alpha})}{2 k_{r}(\boldsymbol{\alpha})}
\end{aligned}
$$

(v) Fifth-order variations:

$$
\begin{aligned}
& \delta^{5} R(T, \boldsymbol{\alpha})=20 \delta^{2} k_{r}(\boldsymbol{\alpha}) \delta^{3} k_{r}(\boldsymbol{\alpha})+10 \delta k_{r}(\boldsymbol{\alpha}) \delta^{4} k_{r}(\boldsymbol{\alpha})+2 k_{r}(\boldsymbol{\alpha}) \delta^{5} k_{r}(\boldsymbol{\alpha}) ; \\
& \Rightarrow \delta^{5} k_{r}(\boldsymbol{\alpha})=\frac{\delta^{5} R(T, \boldsymbol{\alpha})-20 \delta^{2} k_{r}(\boldsymbol{\alpha}) \delta^{3} k_{r}(\boldsymbol{\alpha})-10 \delta k_{r}(\boldsymbol{\alpha}) \delta^{4} k_{r}(\boldsymbol{\alpha})}{2 k_{r}(\boldsymbol{\alpha})}
\end{aligned}
$$

(vi) Sixth-order variations:

$$
\begin{aligned}
0 & =20\left[\delta^{3} k_{r}(\boldsymbol{\alpha})\right]^{2}+30 \delta^{2} k_{r}(\boldsymbol{\alpha}) \delta^{4} k_{r}(\boldsymbol{\alpha})+12 \delta k_{r}(\boldsymbol{\alpha}) \delta^{5} k_{r}(\boldsymbol{\alpha})+2 k_{r}(\boldsymbol{\alpha}) \delta^{6} k_{r}(\boldsymbol{\alpha}) ; \\
& \Rightarrow \delta^{6} k_{r}(\boldsymbol{\alpha})=-\frac{20\left[\delta^{3} k_{r}(\boldsymbol{\alpha})\right]^{2}+30 \delta^{2} k_{r}(\boldsymbol{\alpha}) \delta^{4} k_{r}(\boldsymbol{\alpha})+12 \delta k_{r}(\boldsymbol{\alpha}) \delta^{5} k_{r}(\boldsymbol{\alpha})}{2 k_{r}(\boldsymbol{\alpha})}
\end{aligned}
$$


The G-differentiation of the relation provided in Equation (72) would provide the means for obtaining the expression of $\delta^{6} k_{r}(\boldsymbol{\alpha})$, and so on. Thus, if the variations $\delta^{5} R(T, \boldsymbol{\alpha})$, $n=1, \ldots, 5$, are known, then the expressions of the variations $\delta^{n} k_{r}(\alpha)$ can be obtained successively for $n=1,2, \ldots$, for arbitrarily large $n$.

The exact expressions of the variations $\delta^{n} R(T, \boldsymbol{\alpha}), n=1, \ldots, 4$, can be obtained most efficiently by applying the 4th-CASAM-N developed by Cacuci [1], as will be exemplified in the remainder of this work. Thus, the total first-order sensitivity of $R(T, \boldsymbol{\alpha})$ with respect to parameter variations $\delta \boldsymbol{\alpha}$, is provided by the first-order Gateaux- (G-)differential $\delta R\left(T^{0} ; \boldsymbol{\alpha}^{0} ; \delta T ; \delta \boldsymbol{\alpha}\right)$, which is obtained by applying the definition of the G-differential to Equation (66), which yields the following expression:

$$
\begin{aligned}
& \delta R\left(T^{0} ; \boldsymbol{\alpha}^{0} ; \delta T ; \delta \boldsymbol{\alpha}\right) \triangleq\left\{\frac{d}{d \varepsilon} \int_{0}^{\ell^{0}+\varepsilon \delta \ell}\left(k_{0}^{0}+\varepsilon \delta k_{0}\right)^{2}\left[1+\left(\beta^{0}+\varepsilon \delta \beta\right)\left(T^{0}+\varepsilon \delta T\right)\right]^{2}\right. \\
& \left.\times \delta\left[x-\left(x_{r}^{0}+\varepsilon \delta x_{r}\right)\right] d x\right\}_{\mathcal{\varepsilon}=0} \triangleq\left\{\delta R\left(T^{0} ; \boldsymbol{\alpha}^{0} ; \delta \boldsymbol{\alpha}\right)\right\}_{\text {dir }}+\left\{\delta R\left(T^{0} ; \boldsymbol{\alpha}^{0} ; \delta T\right)\right\}_{\text {ind }} .
\end{aligned}
$$

The direct-effect term $\left\{\delta k_{r}\left(T^{0} ; \boldsymbol{\alpha}^{0} ; \delta \boldsymbol{\alpha}\right)\right\}_{\text {dir }}$, defined in Equation (73), depends only on parameter variations and has the following expression:

$$
\begin{aligned}
& \left\{\delta R\left(T^{0} ; \boldsymbol{\alpha}^{0} ; \delta \boldsymbol{\alpha}\right)\right\}_{d i r} \triangleq\left(\delta k_{0}\right)\left\{2 k_{0} \int_{0}^{\ell}[1+\beta T(x)]^{2} \delta\left(x-x_{r}\right) d x\right\}_{\boldsymbol{\alpha}^{0}} \\
& +(\delta \beta)\left\{2 k_{0}^{2} \int_{0}^{\ell}[1+\beta T(x)] T(x) \delta\left(x-x_{r}\right) d x\right\}_{\boldsymbol{\alpha}^{0}} \\
& -\left(\delta x_{r}\right)\left\{k_{0}^{2} \int_{0}^{\ell}[1+\beta T(x)]^{2} \delta^{\prime}\left(x-x_{r}\right) d x\right\}_{\boldsymbol{\alpha}^{0}} .
\end{aligned}
$$

The indirect-effect term $\left\{\delta R\left(T^{0} ; \boldsymbol{\alpha}^{0} ; \delta T\right)\right\}_{\text {ind }}$, defined in Equation (73), depends only on variations $\delta T(x)$ in the state function $T(x)$ and has the following expression:

$$
\left\{\delta R\left(T^{0} ; \boldsymbol{\alpha}^{0} ; \delta T\right)\right\}_{\text {ind }} \triangleq\left\{2 k_{0}^{2} \beta \int_{0}^{\ell}[1+\beta T(x)] \delta T(x) \delta\left(x-x_{r}\right) d x\right\}_{\boldsymbol{\alpha}^{0}} .
$$

The direct-effect term defined by Equation (74) can be evaluated immediately by replacing the expression provided in Equation (15) for the state function $T(x)$; in practice, this direct-effect term is computed numerically, by using the numerically computed values for $T(x)$.

The indirect-effect term defined by Equation (75) can be evaluated only after having determined the function $\delta T(x)$, which is the solution of the 1st-LVSS represented by Equations (26)-(28). The alternative expression for the indirect-effect term will be obtained in terms of a first-level adjoint sensitivity system (1st-LASS), which is adjoint to the 1stLVSS, has a source term that corresponds to Equation (75), and is constructed by applying the principles of the 4th-CASAM-N just as was conducted in Section 3.1.1, namely:

1. Form the inner product of Equation (26) with a yet undefined function $a^{(1)}(x) \in$ $H_{1}(\Omega)$ and integrate the resulting equations twice by parts to transfer the differential operations from $\delta T(x)$ to $a^{(1)}(x)$, to obtain the following relation: 


$$
\begin{aligned}
& \left\{\int_{0}^{\ell} a^{(1)}(x) \frac{d^{2}}{d x^{2}}[k(T) \delta T(x)] d x\right\}_{\boldsymbol{\alpha}^{0}}=\left\{\int_{0}^{\ell} a^{(1)}(x) s_{V}^{(1)}(T ; \boldsymbol{\alpha} ; \delta \boldsymbol{\alpha}) d x\right\}_{\boldsymbol{\alpha}^{0}} \\
& =\left\{a^{(1)}(x) \frac{d}{d x}\left[k\left(T^{0}\right) \delta T(x)\right]-\delta T(x) k\left(T^{0}\right) \frac{d a^{(1)}(x)}{d x}\right\}_{x=0}^{x=\ell} \\
& +\left\{\int_{0}^{\ell} \delta T(x)\left[k(T) \frac{d^{2} a^{(1)}(x)}{d x^{2}}\right] d x\right\}_{\boldsymbol{\alpha}^{0}} .
\end{aligned}
$$

2. Using the relations that constitute the 1st-LVSS and the 1st-LASS together with Equation (75) in Equation (76) transforms the latter relation into the following form:

$$
\begin{aligned}
& \left\{\delta R\left(T^{0} ; \boldsymbol{\alpha}^{0} ; \delta T\right)\right\}_{\text {ind }}=\left\{\int_{0}^{\ell} a^{(1)}(x) s_{V}^{(1)}(T ; \boldsymbol{\alpha} ; \delta \boldsymbol{\alpha}) d x\right\}_{\boldsymbol{\alpha}^{0}} \\
& -(\delta \ell)\left\{\left[k(T) \frac{d T(x)}{d x} \frac{d a^{(1)}(x)}{d x}\right]_{x=\ell}\right\}_{\boldsymbol{\alpha}^{0}}
\end{aligned}
$$

where the 1st-level adjoint function $a^{(1)}(x)$ is the solution of the following 1st-LASS:

$$
\begin{aligned}
\frac{d^{2} a^{(1)}(x)}{d x^{2}}= & \left\{2 k_{0} \beta \delta\left(x-x_{r}\right)\right\}_{\alpha^{0}}, 0<x<\ell^{0} ; \\
& a^{(1)}(x)=0, \text { at } x=\ell^{0} ; \\
& \frac{d a^{(1)}(x)}{d x}=0, \text { at } x=0 .
\end{aligned}
$$

Adding the expressions obtained in Equations (74) and (77) yields the following expression for the total 1st-order G-differential $\delta R\left(T^{0} ; \boldsymbol{\alpha}^{0} ; \delta T ; \delta \boldsymbol{\alpha}\right)$ :

$$
\begin{aligned}
& \delta R\left(T^{0} ; \boldsymbol{\alpha}^{0} ; \delta T ; \delta \boldsymbol{\alpha}\right)=\left(\delta k_{0}\right)\left\{2 k_{0} \int_{0}^{\ell}[1+\beta T(x)]^{2} \delta\left(x-x_{r}\right) d x\right\}_{\boldsymbol{\alpha}^{0}} \\
& +(\delta \beta)\left\{2 k_{0}^{2} \int_{0}^{\ell}[1+\beta T(x)] T(x) \delta\left(x-x_{r}\right) d x\right\}_{\boldsymbol{\alpha}^{0}} \\
& -\left(\delta x_{r}\right)\left\{k_{0}^{2} \int_{0}^{\ell^{0}}[1+\beta T(x)]^{2} \delta^{\prime}\left(x-x_{r}\right) d x\right\}_{\boldsymbol{\alpha}^{0}} \\
& +\left\{\int_{0}^{\ell} a^{(1)}(x) s_{V}^{(1)}(T ; \boldsymbol{\alpha} ; \delta \boldsymbol{\alpha}) d x\right\}_{\boldsymbol{\alpha}^{0}}-(\delta \ell)\left\{\left[k(T) \frac{d T(x)}{d x} \frac{d a^{(1)}(x)}{d x}\right]_{x=\ell}\right\}_{\boldsymbol{\alpha}^{0}} \\
& \triangleq \frac{\partial R}{\partial Q}(\delta Q)+\frac{\partial R}{\partial k_{0}}\left(\delta k_{0}\right)+\frac{\partial R}{\partial \beta}(\delta \beta)+\frac{\partial R}{\partial x_{r}}\left(\delta x_{r}\right)+\frac{\partial R}{\partial \ell}(\delta \ell) .
\end{aligned}
$$

Replacing in Equation (81) the expression for $s_{V}^{(1)}(T ; \boldsymbol{\alpha} ; \delta \boldsymbol{\alpha})$ from Equation (29) and identifying the quantities that multiply the respective parameter variations yields the following expressions for the 1st-order sensitivities of the response $R(T ; \boldsymbol{\alpha})$ with respect to the model parameters:

$$
\begin{gathered}
\frac{\partial R}{\partial Q}=-\left\{\int_{0}^{\ell} a^{(1)}(x) d x\right\}_{\alpha^{0}} \\
\frac{\partial R}{\partial k_{0}}=\left\{2 k_{0} \int_{0}^{\ell}[1+\beta T(x)]^{2} \delta\left(x-x_{r}\right) d x\right\}_{\alpha^{0}}^{\ell}+\left\{\frac{Q}{k_{0}} \int_{0}^{\ell} a^{(1)}(x) d x\right\}_{\alpha^{0}}^{\prime}
\end{gathered}
$$




$$
\begin{gathered}
\frac{\partial R}{\partial \beta}=\left\{2 k_{0}^{2} \int_{0}^{\ell}[1+\beta T(x)] T(x) \delta\left(x-x_{r}\right) d x\right\}_{\alpha^{0}}-\left\{k_{0} \int_{0}^{\ell} a^{(1)}(x) \frac{d}{d x}\left[T(x) \frac{d T(x)}{d x}\right] d x\right\}_{\boldsymbol{\alpha}^{0}} \\
=\left\{2 k_{0}^{2} \int_{0}^{\ell}[1+\beta T(x)] T(x) \delta\left(x-x_{r}\right) d x\right\}_{\alpha^{0}}-\left\{\frac{k_{0}}{2} \int_{0}^{\ell} T^{2}(x) \frac{d^{2} a^{(1)}(x)}{d x^{2}} d x\right\}_{\alpha^{0}}, \\
\frac{\partial R}{\partial x_{r}}=-\left\{k_{0}^{2} \int_{0}^{\ell}[1+\beta T(x)]^{2} \delta^{\prime}\left(x-x_{r}\right) d x\right\}_{\alpha^{0}}, \\
\frac{\partial R}{\partial \ell}=-\left\{\left[k(T) \frac{d T(x)}{d x} \frac{d a^{(1)}(x)}{d x}\right]_{x=\ell}\right\}_{\alpha^{0}}
\end{gathered}
$$

The expression in the second equality on the right side of Equation (84) has been obtained after integrating twice by parts the second term on the right side of this equation and using the corresponding boundary conditions for the functions $T(x)$ and $a^{(1)}(x)$.

Solving Equations (78)-(80) yields the following explicit expression for the 1st-level adjoint function $a^{(1)}(x)$ :

$$
a^{(1)}(x)=\left\{2 k_{0} \beta\left[\left(x-x_{r}\right) H\left(x-x_{r}\right)-\ell+x_{r}\right]\right\}_{\boldsymbol{\alpha}^{0}} .
$$

Using the expressions for $a^{(1)}(x)$ and $T(x)$ in Equations (82)-(86) yields the following closed-form expressions for the 1st-order sensitivities of the response $R(T ; \boldsymbol{\alpha})$ with respect to the model parameters:

$$
\begin{gathered}
\frac{\partial R}{\partial Q}=k_{0} \beta\left(\ell^{2}-x_{r}^{2}\right), \\
\frac{\partial R}{\partial k_{0}}=2 k_{0}+\beta Q\left(\ell^{2}-x_{r}^{2}\right), \\
\frac{\partial R}{\partial \beta}=k_{0} Q\left(\ell^{2}-x_{r}^{2}\right), \\
\frac{\partial R}{\partial x_{r}}=-2 k_{0} \beta Q x_{r}, \\
\frac{\partial R}{\partial \ell}=2 k_{0} \beta Q \ell .
\end{gathered}
$$

The results obtained in Equations (88)-(92) can be used in conjunction with the results obtained in Equations (61)-(65) for the mutual verification that the relation provided in Equation (67) can indeed be used to compute the sensitivities of when the sensitivities of are known and vice versa, as the following equalities demonstrate:

$$
\begin{gathered}
\frac{\partial k_{r}(\boldsymbol{\alpha})}{\partial Q}=\frac{1}{2 k_{r}(\boldsymbol{\alpha})} \frac{\partial R}{\partial Q}=k_{0} \beta \frac{\ell^{2}-x_{r}^{2}}{2 k_{r}(\boldsymbol{\alpha})}, \\
\frac{\partial k_{r}(\boldsymbol{\alpha})}{\partial k_{0}}=\frac{1}{2 k_{r}(\boldsymbol{\alpha})} \frac{\partial R}{\partial k_{0}}=\frac{k_{0}}{k_{r}(\boldsymbol{\alpha})}+\frac{\beta Q\left(\ell^{2}-x_{r}^{2}\right)}{2 k_{r}(\boldsymbol{\alpha})}, \\
\frac{\partial k_{r}(\boldsymbol{\alpha})}{\partial \beta}=\frac{1}{2 k_{r}(\boldsymbol{\alpha})} \frac{\partial R}{\partial \beta}=\frac{k_{0} Q\left(\ell^{2}-x_{r}^{2}\right)}{2 k_{r}(\boldsymbol{\alpha})}, \\
\frac{\partial k_{r}(\boldsymbol{\alpha})}{\partial x_{r}}=\frac{1}{2 k_{r}(\boldsymbol{\alpha})} \frac{\partial R}{\partial x_{r}}=-\frac{k_{0} \beta Q x_{r}}{k_{r}(\boldsymbol{\alpha})} \\
\frac{\partial k_{r}}{\partial \ell}=\frac{1}{2 k_{r}(\boldsymbol{\alpha})} \frac{\partial R}{\partial \ell}=\frac{k_{0} \beta Q \ell}{k_{r}(\boldsymbol{\alpha})} .
\end{gathered}
$$




\subsection{Second-Order Response Sensitivities}

This section will illustrate the application of the principles of the 4th-CASAM-N methodology to derive exact expressions for representative 2nd-order sensitivities corresponding to the 1st-order sensitivities obtained in Equations (82)-(86). Each of the 1st-order sensitivities can be considered to represent a "model response." Consequently, by applying the principles outlined in Section 3.1, above, to each of the 1st-order sensitivities would generate the corresponding 2nd-order sensitivities by using a single (2nd-level) adjoint function. As the illustrative nonlinear heat conduction paradigm model has five (uncertain) parameters, a total of five 2nd-level adjoint functions will be needed to generate all of the 25 second-order sensitivities. The mixed 2 nd-order sensitivities will be generated twice, using distinct adjoint functions; hence, the symmetries inherent to the 2nd-order sensitivities provide an intrinsic verification mechanism for ensuring that the 2 nd-level adjoint functions are accurately computed. Of course, it is not necessary to generate all of the 2nd-order sensitivities: only the ones deemed to be important (based on the relative magnitudes of the 1st-order sensitivities) would need to be generated. These concepts will be illustrated in this and subsequent sections.

As indicated in Equation (82), the expression of the 1st-order sensitivity $\partial R / \partial Q$ involves only the first-level adjoint function $a^{(1)}(x)$. Consequently, the principles of the 4th-CASAM-N methodology indicate that the 2nd-order sensitivities corresponding to $\partial R / \partial Q$ will be determined in terms of a 2nd-level adjoint function, which will comprise a single component that will correspond to the variational function $\delta a^{(1)}(x)$. The expression of the 1st-order sensitivity $\partial R / \partial x_{r}$ obtained in Equation (85) involves only the forward state function $T(x)$. Consequently, the principles of the 4th-CASAM-N methodology indicate that the 2nd-order sensitivities corresponding to $\partial R / \partial x_{r}$ will be determined in terms of a 2nd-level adjoint function, which will (also) comprise a single component that will correspond to the variational function $\delta T(x)$. On the other hand, the expressions obtained in Equations (83), (84) and (86) indicate that those 1st-order sensitivities involve both $a^{(1)}(x)$ and $T(x)$. Consequently, the principles of the 4th-CASAM-N methodology indicate that the 2nd-order sensitivities corresponding to the 1st-order sensitivities obtained in Equations (83), (84) and (86) will involve 2nd-level adjoint functions which will each comprise two-components (as opposed to a single component); one component corresponding to $\delta T(x)$ and the second component corresponding to $\delta a^{(1)}(x)$.

Section 3.2.1 will illustrate the application of the 4th-CASAM-N methodology to determine the exact expressions of the 2nd-order sensitivities that correspond to $\partial R / \partial Q$, thereby illustrating the procedure involved when the 2nd-level adjoint function comprises just one component. Section 3.2.2 will illustrate the application of the 4th-CASAM-N methodology to determine the exact expressions of the 2nd-order sensitivities that correspond to $\partial R / \partial k_{0}$, thereby illustrating the procedure involved when the 2nd-level adjoint function comprises two components.

\subsubsection{Second-Order Sensitivities Corresponding to $\partial R / \partial Q$}

The application of the 4 th-CASAM-N to compute efficiently and exactly second-order sensitivities will be illustrated in this subsection by considering the 1st-order sensitivity $\partial R / \partial Q$, the expression of which has been obtained in Equation (82). The sensitivity $\partial R / \partial Q$ is representative of the procedure involved when applying the 4th-CASAM-N to a 1st-order sensitivity that involves a single state function, which in this case is the 1st-level adjoint function $a^{(1)}(x)$.

The second-order sensitivities corresponding to $\partial R / \partial k_{0}$ are obtained by determining the G-differential of Equation (83), which has, by definition, the following expression:

$$
\left\{\delta\left[\frac{\partial R}{\partial Q}\right]\right\}_{\alpha^{0}} \triangleq-\left\{\frac{d}{d \varepsilon}\left[\int_{0}^{\ell^{0}+\varepsilon \delta \ell}\left[a^{(1), 0}(x)+\varepsilon \delta a^{(1)}(x)\right] d x\right]\right\}_{\varepsilon=0} \triangleq\{\delta[\partial R / \partial Q]\}_{d i r}+\{\delta[\partial R / \partial Q]\}_{\text {ind }}
$$


The direct-effect term $\{\delta[\partial R / \partial Q]\}_{\text {dir }}$ is defined to depend only on parameter variations and, in the case of Equation (98), stems only from the derivative with respect to $\varepsilon$ of the upper limit of integration, i.e.,

$$
\left\{\delta\left[\partial R / \partial k_{0}\right]\right\}_{d i r} \triangleq-(\delta \ell)\left\{a^{(1)}\left(x=\ell^{0}\right)\right\}_{\alpha^{0}}=0 .
$$

As indicated in Equation (99), the direct-effect term vanishes because of the boundary condition satisfied by the 1st-level adjoint function $a^{(1)}(x)$ provided in Equation (79). Therefore, the G-differential $\{\delta[\partial R / \partial Q]\}_{\alpha^{0}}$ comprises only the indirect-effect term $\{\delta[\partial R / \partial Q]\}_{\text {ind }}$, which depends only on the variation $\delta a^{(1)}(x)$ and is defined as follows:

$$
\{\delta[\partial R / \partial Q]\}_{\text {ind }} \triangleq-\left\{\int_{0}^{\ell} \delta a^{(1)}(x) d x\right\}_{\boldsymbol{\alpha}^{0}}=\{\delta[\partial R / \partial Q]\}_{\boldsymbol{\alpha}^{0}} .
$$

The indirect-effect term defined by Equation (100) can be evaluated only after having determined the variational function $\delta a^{(1)}(x)$, which is the solution of the system obtained by taking the G-differential of the 1st-LASS defined by Equations (78)-(80), and which has the following expression:

$$
\begin{gathered}
\frac{d^{2}\left[\delta a^{(1)}(x)\right]}{d x^{2}}=\left(\delta k_{0}\right)\left\{2 \beta \delta\left(x-x_{r}\right)\right\}_{\boldsymbol{\alpha}^{0}}+(\delta \beta)\left\{2 k_{0} \delta\left(x-x_{r}\right)\right\}_{\boldsymbol{\alpha}^{0}} \\
-\left(\delta x_{r}\right)\left\{2 k_{0} \beta \delta^{\prime}\left(x-x_{r}\right)\right\}_{\boldsymbol{\alpha}^{0}}, 0<x<\ell^{0} ; \\
\frac{d}{d x}\left[\delta a^{(1)}(x)\right]=0, \text { at } x=0 ; \\
\delta a^{(1)}(x)+(\delta \ell)\left\{\frac{d a^{(1)}(x)}{d x}\right\}_{\boldsymbol{\alpha}^{0}}=0, \text { at } x=\ell^{0} .
\end{gathered}
$$

The system comprising Equations (101)-(103) is actually the 2nd-level variational sensitivity system (2nd-LVSS) for the function $\delta a^{(1)}(x)$. Its solution, $\delta a^{(1)}(x)$, could be used to determine the G-differential $\{\delta[\partial R / \partial Q]\}_{\alpha^{0}}$ shown in Equation (98). As $\delta a^{(1)}(x)$ depends on parameter variations, however, solving repeatedly the 2nd-LVSS for all parameter variations is avoided by constructing a corresponding 2nd-LASS, which would need to be solved only once, as its solution would be independent of parameter variations and would be used to obtain the G-differential $\{\delta[\partial R / \partial Q]\}_{\alpha^{0}}$. As the 2nd-LVSS comprising Equations (101)-(103) has the same structure as the 1st-LVSS comprising Equations (26)-(28), it follows that the 2nd-LASS that corresponds to the 2nd-LVSS comprising Equations (101)-(103) will be constructed by applying the same principles that were applied to construct the 1st-LASS in Section 3.1 when determining the 1 st-order sensitivities of the response $R(T, \boldsymbol{\alpha}) \triangleq k_{r}^{2}$. Thus, the inner product defined in Equation (30) will be used to construct the inner product of Equation (101) with a yet undefined one-component function $a_{Q}^{(2)}(x)$-which will ultimately become the sought-after 2nd-level adjoint function-to obtain the following relation:

$$
\begin{aligned}
& \left\{\int_{0}^{\ell} a_{Q}^{(2)}(x) \frac{d^{2}\left[\delta a^{(1)}(x)\right]}{d x^{2}} d x\right\}_{\alpha^{0}}=\left(\delta k_{0}\right)\left\{2 \beta \int_{0}^{\ell} a_{Q}^{(2)}(x) \delta\left(x-x_{r}\right) d x\right\}_{\alpha^{0}} \\
& +(\delta \beta)\left\{2 k_{0} \int_{0}^{\ell} a_{Q}^{(2)}(x) \delta\left(x-x_{r}\right) d x\right\}_{\alpha^{0}}-\left(\delta x_{r}\right)\left\{2 k_{0} \beta \int_{0}^{\ell} a_{Q}^{(2)}(x) \delta^{\prime}\left(x-x_{r}\right) d x\right\}_{\alpha^{0}} .
\end{aligned}
$$

The superscript " 2 " in the notation $a_{Q}^{(2)}(x)$ indicates " 2 nd-level" while the subscript " $Q$ " indicates that that this 2nd-level adjoint function corresponds to the parameter " $Q$ ". Integrating the left side of Equation (104) twice by parts yields the following relation: 


$$
\begin{gathered}
\left\{\int_{0}^{\ell} a_{Q}^{(2)}(x) \frac{d^{2}\left[\delta a^{(1)}(x)\right]}{d x^{2}} d x\right\}_{\alpha^{0}}=\left\{\int_{0}^{\ell} \delta a^{(1)}(x) \frac{d^{2}\left[a_{Q}^{(2)}(x)\right]}{d x^{2}} d x\right\}_{\alpha^{0}} \\
+\left\{a_{Q}^{(2)}(x) \frac{d}{d x}\left[\delta a^{(1)}(x)\right]-\delta a^{(1)}(x) \frac{d}{d x}\left[a_{Q}^{(2)}(x)\right]\right\}_{x=0}^{x=\ell} .
\end{gathered}
$$

Using the relation provided in Equation (104) and inserting into Equation (105) the boundary conditions provided in Equations (102) and (103) makes it possible to recast Equation (105) into the following form:

$$
\begin{aligned}
& \left\{\int_{0}^{\ell} \delta a^{(1)}(x) \frac{d^{2}\left[a_{Q}^{(2)}(x)\right]}{d x^{2}} d x\right\}_{\alpha^{0}}=\left(\delta k_{0}\right)\left\{2 \beta \int_{0}^{\ell} a_{Q}^{(2)}(x) \delta\left(x-x_{r}\right) d x\right\}_{\alpha^{0}} \\
& +(\delta \beta)\left\{2 k_{0} \int_{0}^{\ell} a_{Q}^{(2)}(x) \delta\left(x-x_{r}\right) d x\right\}_{\alpha^{0}}-\left(\delta x_{r}\right)\left\{2 k_{0} \beta \int_{0}^{\ell} a_{Q}^{(2)}(x) \delta^{\prime}\left(x-x_{r}\right) d x\right\}_{\alpha^{0}} \\
& -\left\{a_{Q}^{(2)}(x) \frac{d}{d x}\left[\delta a^{(1)}(x)\right]+(\delta \ell) \frac{d a^{(1)}(x)}{d x} \frac{d}{d x}\left[a_{Q}^{(2)}(x)\right]\right\}_{x=\ell}-\left\{\delta a^{(1)}(x) \frac{d}{d x}\left[a_{Q}^{(2)}(x)\right]\right\}_{x=0} .
\end{aligned}
$$

The left side of Equation (106) is now required to represent the G-differential defined in Equation (100). Furthermore, the definition of the 2nd-level adjoint function $a_{Q}^{(2)}(x)$ is completed by requiring that it satisfy boundary conditions, which would eliminate the unknown boundary terms from Equation (106). Imposing these requirements yields the following 2nd-LASS for $a_{Q}^{(2)}(x)$ :

$$
\begin{gathered}
\frac{d^{2}}{d x^{2}} a_{Q}^{(2)}(x)=-1, \quad 0<x<\ell^{0} ; \\
a_{Q}^{(2)}(x)=0, \text { at } x=\ell^{0} ; \\
\frac{d\left[a_{Q}^{(2)}(x)\right]}{d x}=0, \text { at } x=0 .
\end{gathered}
$$

Recalling Equation (100) and implementing the relations represented by the 2nd-LASS into Equation (106) transforms the latter relation into the following form:

$$
\begin{aligned}
& \{\delta[\partial R / \partial Q]\}_{\text {ind }}=-(\delta \ell)\left\{\frac{d a^{(1)}(x)}{d x} \frac{d}{d x}\left[a_{Q}^{(2)}(x)\right]\right\}_{x=\ell}+\left(\delta k_{0}\right)\left\{2 \beta \int_{0}^{\ell} a_{Q}^{(2)}(x) \delta\left(x-x_{r}\right) d x\right\}_{\alpha^{0}} \\
& +(\delta \beta)\left\{2 k_{0} \int_{0}^{\ell} a_{Q}^{(2)}(x) \delta\left(x-x_{r}\right) d x\right\}_{\alpha^{0}}-\left(\delta x_{r}\right)\left\{2 k_{0} \beta \int_{0}^{\ell} a_{Q}^{(2)}(x) \delta^{\prime}\left(x-x_{r}\right) d x\right\}_{\alpha^{0}} .
\end{aligned}
$$

Identifying the quantities that multiply the various parameter variations in Equation (110) yields the following expressions for the corresponding 2nd-order sensitivities:

$$
\begin{gathered}
\frac{\partial^{2} R}{\partial Q \partial Q} \equiv 0, \\
\frac{\partial^{2} R}{\partial k_{0} \partial Q}=2 \beta \int_{0}^{\ell} a_{Q}^{(2)}(x) \delta\left(x-x_{r}\right) d x, \\
\frac{\partial^{2} R}{\partial \beta \partial Q}=2 k_{0} \int_{0}^{\ell} a_{Q}^{(2)}(x) \delta\left(x-x_{r}\right) d x,
\end{gathered}
$$




$$
\begin{gathered}
\frac{\partial^{2} R}{\partial x_{r} \partial Q}=-2 k_{0} \beta \int_{0}^{\ell} a_{Q}^{(2)}(x) \delta^{\prime}\left(x-x_{r}\right) d x, \\
\frac{\partial^{2} R}{\partial \ell \partial Q}=-\left\{\frac{d a^{(1)}(x)}{d x} \frac{d}{d x}\left[a_{Q}^{(2)}(x)\right]\right\}_{x=\ell} .
\end{gathered}
$$

The expressions on the right sides of Equations (112)-(115) are to be evaluated at the nominal values of the respective parameters and state functions but the notation \{\}$_{\alpha^{0}}$ has been omitted for simplicity.

The expressions for the 2nd-order sensitivities obtained in Equations (112)-(115) can be evaluated inexpensively, using quadrature formulas, after having solved the 2nd-LASS once to obtain the 2nd-level adjoint function. Thus, solving the 2nd-LASS is the sole "large-scale" computation needed in order to compute the respective sensitivities. In contradistinction, using forward methods would have required at least as many "largescale" computations as there are model parameters.

Solving Equations (107)-(109) yields the following explicit expression for the 2nd-level adjoint function $a_{Q}^{(2)}(x)$ :

$$
a_{Q}^{(2)}(x)=\left\{\left(\ell^{2}-x^{2}\right) / 2\right\}_{\alpha^{0}} .
$$

Using the expressions for $a^{(1)}(x)$ and $a_{Q}^{(2)}(x)$ in Equations (112)-(115) yields the following closed-form expressions for the respective 2nd-order sensitivities:

$$
\begin{gathered}
\frac{\partial^{2} R}{\partial k_{0} \partial Q}=\beta\left(\ell^{2}-x_{r}^{2}\right), \\
\frac{\partial^{2} R}{\partial \beta \partial Q}=k_{0}\left(\ell^{2}-x_{r}^{2}\right), \\
\frac{\partial^{2} R}{\partial x_{r} \partial Q}=-2 k_{0} \beta x_{r}, \\
\frac{\partial^{2} R}{\partial \ell \partial Q}=2 k_{0} \beta \ell .
\end{gathered}
$$

Of course, the above closed-form expressions are for verification purposes; in practice, the values of the respective sensitivities are computed numerically using Equations (112)-(115), as has been mentioned in the foregoing.

\subsubsection{Second-Order Sensitivities Corresponding to $\partial R / \partial k_{0}$}

The application of the 4th-CASAM-N to compute efficiently and exactly second-order sensitivities will be illustrated in this subsection by considering the 1st-order sensitivity $\partial R / \partial k_{0}$, the expression of which has been obtained in Equation (83). The sensitivity $\partial R / \partial k_{0}$ is representative of the procedure involved when applying the 4th-CASAM-N to a 1st-order sensitivity that involves both the original forward function $T(x)$ and the 1st-level adjoint function $a^{(1)}(x)$.

The second-order sensitivities corresponding to $\partial R / \partial k_{0}$ are obtained by determining the G-differential of Equation (83), which has, by definition, the following expression:

$$
\begin{aligned}
& \left\{\delta\left[\frac{\partial R}{\partial k_{0}}\right]\right\}_{\alpha^{0}} \triangleq\left\{\frac{d}{d \varepsilon}\left[2\left(k_{0}^{0}+\varepsilon \delta k_{0}\right) \int_{0}^{\ell^{0}+\varepsilon \delta \ell}\left[1+\left(\beta^{0}+\varepsilon \delta \beta\right)\left(T^{0}+\varepsilon \delta T\right)\right]^{2} \delta\left[x-\left(x_{r}^{0}+\varepsilon \delta x_{r}\right)\right] d x\right]\right\}_{\varepsilon=0} \\
& +\left\{\frac{d}{d \varepsilon}\left[\frac{Q^{0}+\varepsilon \delta Q}{k_{0}^{0}+\varepsilon \delta k_{0}} \int_{0}^{\ell^{0}+\varepsilon \delta \ell}\left(a^{(1), 0}+\varepsilon \delta a^{(1)}\right) d x\right]\right\}_{\varepsilon=0} \triangleq\left\{\delta\left[\partial R / \partial k_{0}\right]\right\}_{d i r}+\left\{\delta\left[\partial R / \partial k_{0}\right]\right\}_{\text {ind }^{\prime}}
\end{aligned}
$$


where the direct-effect term $\left\{\delta\left[\partial R / \partial k_{0}\right]\right\}_{d i r}$ depends only on parameter variations and is defined as follows:

$$
\begin{aligned}
& \left\{\delta\left[\partial R / \partial k_{0}\right]\right\}_{d i r} \triangleq(\delta Q)\left\{\frac{1}{k_{0}} \int_{0}^{\ell} a^{(1)}(x) d x\right\}_{\alpha^{0}} \\
& -\left(\delta k_{0}\right)\left\{\frac{Q}{k_{0}^{2}} \int_{0}^{\ell} a^{(1)}(x) d x-2 \int_{0}^{\ell}[1+\beta T(x)]^{2} \delta\left(x-x_{r}\right) d x\right\}_{\alpha^{0}} \\
& +(\delta \beta)\left\{4 k_{0} \int_{0}^{\ell}[1+\beta T(x)] T(x) \delta\left(x-x_{r}\right) d x\right\}_{\alpha^{0}} \\
& -\left(\delta x_{r}\right)\left\{2 k_{0}^{\ell_{0}^{0}}[1+\beta T(x)]^{2} \delta^{\prime}\left(x-x_{r}\right) d x\right\}_{\alpha^{0}},
\end{aligned}
$$

and where the indirect-effect term $\left\{\delta\left[\partial R / \partial k_{0}\right]\right\}_{\text {ind }}$ depends only on variations $\delta T(x)$ and $\delta a^{(1)}(x)$, and is defined as follows:

$$
\left\{\delta\left[\partial R / \partial k_{0}\right]\right\}_{\text {ind }} \triangleq\left\{2 k_{0}^{2} \beta \int_{0}^{\ell}[1+\beta T(x)] \delta T(x) \delta\left(x-x_{r}\right) d x\right\}_{\boldsymbol{\alpha}^{0}}+\left\{\frac{Q}{k_{0}} \int_{0}^{\ell} \delta a^{(1)}(x) d x\right\}_{\boldsymbol{\alpha}^{0}} .
$$

The direct-effect term defined by Equation (122) can be evaluated immediately, as the state functions $T(x)$ and $a^{(1)}(x)$ are available already; in practice, this direct-effect term is computed numerically. However, the indirect-effect term defined by Equation (123) can be evaluated only after having determined the functions $\delta T(x)$ and $\delta a^{(1)}(x)$. The function $\delta T(x)$ is the solution of the 1st-LVSS represented by Equations (26)-(28). The function $\delta a^{(1)}(x)$ is the solution of the system obtained by taking the G-differential of the 1st-LASS defined by Equations (78)-(80), which has the following expression:

$$
\begin{gathered}
\frac{d^{2}\left[\delta a^{(1)}(x)\right]}{d x^{2}}=\left(\delta k_{0}\right)\left\{2 \beta \delta\left(x-x_{r}\right)\right\}_{\boldsymbol{\alpha}^{0}}+(\delta \beta)\left\{2 k_{0} \delta\left(x-x_{r}\right)\right\}_{\boldsymbol{\alpha}^{0}} \\
-\left(\delta x_{r}\right)\left\{2 k_{0} \beta \delta^{\prime}\left(x-x_{r}\right)\right\}_{\boldsymbol{\alpha}^{0}}, \quad 0<x<\ell^{0} ; \\
\frac{d}{d x}\left[\delta a^{(1)}(x)\right]=0, \text { at } x=0 ; \\
\delta a^{(1)}(x)+(\delta \ell)\left\{\frac{d a^{(1)}(x)}{d x}\right\}_{\boldsymbol{\alpha}^{0}}=0, \text { at } x=\ell^{0} .
\end{gathered}
$$

Concatenating the 1st-LVSS and the G-differentiated 1st-LASS yields the following 2ndlevel variational sensitivity system (2nd-LVSS), comprising Equations (127) and (128) below, for obtaining the 2 nd-level variational function $\mathbf{v}^{(2)}(2 ; \mathbf{x})$, which is also defined below:

$$
\begin{gathered}
\left\{\mathbf{V M}^{(2)} \mathbf{V}^{(2)}(2 ; \mathbf{x})\right\}_{\boldsymbol{\alpha}^{0}}=\left\{\mathbf{Q}_{V}^{(2)}(\boldsymbol{\alpha} ; \delta \boldsymbol{\alpha})\right\}_{\boldsymbol{\alpha}^{0^{\prime}}} \\
\mathbf{x} \in \Omega_{x} \\
\mathbf{B}_{V}^{(2)}(2 ; \boldsymbol{\alpha} ; \delta \boldsymbol{\alpha}) \triangleq\left(\begin{array}{c}
\mathbf{b}_{V}^{(2)}(1 ; \boldsymbol{\alpha} ; \delta \boldsymbol{\alpha}) \\
\mathbf{b}_{V}^{(2)}(2 ; \boldsymbol{\alpha} ; \delta \boldsymbol{\alpha})
\end{array}\right)=\left(\begin{array}{c}
0 \\
0
\end{array}\right)
\end{gathered}
$$

where

$$
\begin{aligned}
& \mathbf{V}^{(2)}(2 ; x) \triangleq\left(\begin{array}{c}
v^{(2)}(1 ; x) \\
v^{(2)}(2 ; x)
\end{array}\right) \triangleq\left(\begin{array}{c}
\delta T(x) \\
\delta \psi^{(1)}(x)
\end{array}\right) ; \mathbf{Q}_{V}^{(2)}(\boldsymbol{\alpha} ; \delta \boldsymbol{\alpha}) \triangleq\left(\begin{array}{c}
q_{V}^{(2)}(1 ; \boldsymbol{\alpha} ; \delta \boldsymbol{\alpha}) \\
q_{V}^{(2)}(2 ; \boldsymbol{\alpha} ; \delta \boldsymbol{\alpha})
\end{array}\right) ; \\
& \mathbf{V M}^{(2)}(2 \times 2 ; x) \triangleq\left(\begin{array}{cc}
\frac{d^{2}}{d x^{2}}\{k(T)[]\} & 0 \\
0 & \frac{d^{2}[]}{d x^{2}}
\end{array}\right)
\end{aligned}
$$


with

$$
\begin{gathered}
q_{V}^{(2)}(1 ; \boldsymbol{\alpha} ; \delta \boldsymbol{\alpha}) \triangleq s_{V}^{(1)}(T ; \boldsymbol{\alpha} ; \delta \boldsymbol{\alpha}) \triangleq-(\delta Q)+\left(\delta k_{0}\right) \frac{Q}{k_{0}}-(\delta \beta) k_{0} \frac{d}{d x}\left[T(x) \frac{d T(x)}{d x}\right] \\
q_{V}^{(2)}(2 ; \boldsymbol{\alpha} ; \delta \boldsymbol{\alpha}) \triangleq\left(\delta k_{0}\right) 2 \beta \delta\left(x-x_{r}\right)+(\delta \beta) 2 k_{0} \delta\left(x-x_{r}\right)-\left(\delta x_{r}\right) 2 k_{0} \beta \delta^{\prime}\left(x-x_{r}\right) ; \\
\mathbf{b}_{V}^{(2)}(1 ; \boldsymbol{\alpha} ; \delta \boldsymbol{\alpha}) \triangleq\left(\begin{array}{c}
\{d[\delta T(x)] / d x\}_{x=0} \\
\{\delta T(x)\}_{x=\ell^{0}}+(\delta \ell)\{d T(x) / d x\}_{x=\ell^{0}}
\end{array}\right) ; \\
\left\{d\left[\delta \psi^{(1)}(x)\right] / d x\right\}_{x=0} \\
\mathbf{b}_{V}^{(2)}(2 ; \boldsymbol{\alpha} ; \delta \boldsymbol{\alpha}) \triangleq\left(\begin{array}{c}
\left\{\psi^{(1)}(x)\right\}_{x=\ell^{0}}+(\delta \ell)\left\{d \psi^{(1)}(x) / d x\right\}_{x=\ell^{0}}
\end{array}\right) ;
\end{gathered}
$$

To distinguish vectors from matrices, two capital bold letters were used (and will henceforth be used) to denote matrices, as in the case of "the second-level variational matrix" $\mathbf{V M}^{(2)}\left[2 \times 2 ; \mathbf{u}^{(2)}(\mathbf{x}) ; \boldsymbol{\alpha}\right]$. The "2nd-level" is indicated by the superscript " $(2)$ ". Subsequently in this work, levels higher than the second will also be indicated by a corresponding superscript attached to the appropriate (block-) vectors and/or (block-) matrices. The argument " $2 \times 2$ ", which appears in the list of arguments of $\mathbf{V M}^{(2)}(2 \times 2 ; \boldsymbol{\alpha})$, indicates that this matrix is a $2 \times 2$-dimensional matrix.

The need for solving the 2nd-LVSS is circumvented by deriving an alternative expression for the indirect-effect term defined in Equation (123), in which the function $\mathbf{V}^{(2)}(2 ; \mathbf{x})$ is replaced by a 2nd-level adjoint function that is independent of variations in the model parameter and state functions. This 2 nd-level adjoint function will satisfy a 2 nd-level adjoint sensitivity system (2nd-LASS) which will be constructed by using the 2nd-LVSS as the starting point and following the same principles as outlined in Section 3.1. The 2nd-LASS will be constructed in a Hilbert space, which will be denoted as $H_{2}\left(\Omega_{x}\right)$ and which is comprised of element vectors of the same form as $\mathbf{V}^{(2)}(2 ; \mathbf{x})$; a generic vector in $H_{2}\left(\Omega_{x}\right)$ is a two-component column vector of the form $\Psi^{(2)}(2 ; x) \triangleq\left[\psi^{(2)}(1 ; x), \psi^{(2)}(2 ; x)\right]^{\dagger} \in H_{2}\left(\Omega_{x}\right)$. The inner product of two vectors $\Psi^{(2)}(2 ; x) \triangleq\left[\psi^{(2)}(1 ; x), \psi^{(2)}(2 ; x)\right]^{\dagger} \in H_{2}\left(\Omega_{x}\right)$ and $\boldsymbol{\Phi}^{(2)}(2 ; x) \triangleq\left[\varphi^{(2)}(1 ; x), \varphi^{(2)}(2 ; x)\right]^{\dagger} \in H_{2}\left(\Omega_{x}\right)$ in the Hilbert space $H_{2}\left(\Omega_{x}\right)$ will be denoted as $\left\langle\Psi^{(2)}(2 ; x), \boldsymbol{\Phi}^{(2)}(x)\right\rangle_{2}$ and defined as follows:

$$
\left\langle\mathbf{\Psi}^{(2)}(2 ; \mathbf{x}), \boldsymbol{\Phi}^{(2)}(\mathbf{x})\right\rangle_{2} \triangleq \sum_{i=1}^{2}\left\langle\psi^{(2)}(i ; \mathbf{x}), \varphi^{(2)}(i ; \mathbf{x})\right\rangle_{1}=\sum_{i=1}^{2}\left\{\int_{0}^{\ell} \psi^{(2)}(i ; \mathbf{x}), \varphi^{(2)}(i ; \mathbf{x}) d x\right\}_{\boldsymbol{\alpha}^{0}} .
$$

The inner product defined in Equation (133) is continuous in $\alpha$ in a neighborhood of $\alpha^{0}$. Using the definition of the inner product defined in Equation (133), construct the inner product of Equation (127) with a vector $\mathbf{A}_{k}^{(2)}(2 ; x) \triangleq\left[a_{k}^{(2)}(1 ; x), a_{k}^{(2)}(2 ; x)\right]^{\dagger} \in H_{2}\left(\Omega_{x}\right)$ to obtain the following relation:

$$
\left\{\left\langle\mathbf{A}_{k}^{(2)}(2 ; x), \mathbf{V M}^{(2)}(2 \times 2 ; \boldsymbol{\alpha}) \mathbf{V}^{(2)}(2 ; x)\right\rangle_{2}\right\}_{\boldsymbol{\alpha}^{0}}=\left\{\left\langle\mathbf{A}_{k}^{(2)}(2 ; x), \mathbf{Q}_{V}^{(2)}(2 ; \boldsymbol{\alpha} ; \delta \boldsymbol{\alpha})\right\rangle_{2}\right\}_{\boldsymbol{\alpha}^{0}} .
$$

The notation chosen for the vector $\mathbf{A}_{k}^{(2)}(2 ; x)$ indicates the following characteristics of this vector: (i) the bold letter $\mathbf{A}$ indicates that this quantity is a vector; (ii) the superscript " 2 " indicates that this quantity is a "second-level" function; (iii) the subscript " $k$ " indicates that this quantity corresponds to the 1 st-order sensitivity $\partial R / \partial k_{0}$ of the response with respect to the parameter $k_{0}$; (iv) the digit " 2 " in the argument of $\mathbf{A}_{k}^{(2)}(2 ; x)$ indicates that this vector has two components; (v) the letter " $x$ " in the argument of $\mathbf{A}_{k}^{(2)}(2 ; x)$ indicates that the two components of this vector are functions of the independent variable " $x$ ". 
In component form, Equation (134) has the following expression:

$$
\begin{aligned}
& \left\{\int_{0}^{\ell} a_{k}^{(2)}(1 ; x) \frac{d^{2}}{d x^{2}}[k(T) \delta T(x)] d x\right\}_{\boldsymbol{\alpha}^{0}}+\left\{\int_{0}^{\ell} a_{k}^{(2)}(2 ; x) \frac{d^{2}\left[\delta a^{(1)}(x)\right]}{d x^{2}} d x\right\}_{\boldsymbol{\alpha}^{0}} \\
& =\left\{\int_{0}^{\ell} a_{k}^{(2)}(1 ; x) q_{V}^{(2)}(1 ; \boldsymbol{\alpha} ; \delta \boldsymbol{\alpha}) d x\right\}_{\boldsymbol{\alpha}^{0}}+\left\{\int_{0}^{\ell} a_{k}^{(2)}(2 ; x) q_{V}^{(2)}(2 ; \boldsymbol{\alpha} ; \delta \boldsymbol{\alpha}) d x\right\}_{\boldsymbol{\alpha}^{0}} .
\end{aligned}
$$

The inner product on the left side of Equation (134) is now further transformed by using the definition of the adjoint operator to obtain, after integrating twice by parts over the independent variable $x$, the following relation:

$$
\begin{aligned}
& \left\{\left\langle\mathbf{A}_{k}^{(2)}(2 ; x), \mathbf{V M}^{(2)}(2 \times 2 ; \boldsymbol{\alpha}) \mathbf{V}^{(2)}(2 ; x)\right\rangle_{2}\right\}_{\boldsymbol{\alpha}^{0}} \\
& =\left\{\left\langle\mathbf{V}^{(2)}(2 ; x), \mathbf{A M}^{(2)}(2 \times 2 ; \boldsymbol{\alpha}) \mathbf{A}_{k}^{(2)}(2 ; x)\right\rangle_{2}\right\}_{\boldsymbol{\alpha}^{0}}+\left\{\left[P^{(2)}\left(\mathbf{A}_{k}^{(2)} ; \mathbf{V}^{(2)} ; \boldsymbol{\alpha}\right)\right]_{\partial \Omega_{x}}\right\}_{\boldsymbol{\alpha}^{0}}
\end{aligned}
$$

In Equation (136), the adjoint (matrix-valued) operator $\mathbf{A M}^{(2)}(2 \times 2 ; \boldsymbol{\alpha})$ has the following form:

$$
\mathbf{A M}^{(2)}(2 \times 2 ; \boldsymbol{\alpha})=\left(\begin{array}{cc}
k(T) \frac{d^{2}[]}{d x^{2}} & 0 \\
0 & \frac{d^{2}[]}{d x^{2}}
\end{array}\right)
$$

while the quantity $\left\{\left[P^{(2)}\left(\mathbf{A}^{(2)} ; \mathbf{V}^{(2)} ; \boldsymbol{\alpha}\right)\right]_{\partial \Omega_{x}}\right\}_{\alpha^{0}}$ denotes the corresponding bilinear concomitant on the domain's boundary, evaluated at the nominal values for the parameters and respective state functions, and having the following expression:

$$
\begin{aligned}
& \left\{\left[P^{(2)}\left(\mathbf{A}_{k}^{(2)} ; \mathbf{V}^{(2)} ; \boldsymbol{\alpha}\right)\right]_{\partial \Omega_{x}}\right\}_{\boldsymbol{\alpha}^{0}}=\left\{a_{k}^{(2)}(1 ; x) \frac{d}{d x}\left[k\left(T^{0}\right) \delta T(x)\right]-\delta T(x) k\left(T^{0}\right) \frac{d}{d x}\left[a_{k}^{(2)}(1 ; x)\right]\right\}_{x=0}^{x=\ell} \\
& +\left\{a_{k}^{(2)}(2 ; x) \frac{d}{d x}\left[\delta a^{(1)}(x)\right]-\delta a^{(1)}(x) \frac{d}{d x}\left[a_{k}^{(2)}(2 ; x)\right]\right\}_{x=0}^{x=\ell} .
\end{aligned}
$$

Inserting into Equation (138) the 2nd-LVSS's boundary conditions provided in Equation (128) reduces the bilinear concomitant $\left\{\left[P^{(2)}\left(\mathbf{A}_{k}^{(2)} ; \mathbf{V}^{(2)} ; \boldsymbol{\alpha}\right)\right]_{\partial \Omega_{x}}\right\}_{\alpha^{0}}$ to the following expression:

$$
\begin{aligned}
& \left\{\left[P^{(2)}\left(\mathbf{A}_{k}^{(2)} ; \mathbf{V}^{(2)} ; \boldsymbol{\alpha}\right)\right]_{\partial \Omega_{x}}\right\}_{\boldsymbol{\alpha}^{0}} \\
& =\left\{a_{k}^{(2)}(1 ; x) \frac{d}{d x}\left[k\left(T^{0}\right) \delta T(x)\right]+\left[(\delta \ell) \frac{d T(x)}{d x}\right] k\left(T^{0}\right) \frac{d a_{k}^{(2)}(1 ; x)}{d x}\right\}_{x=\ell^{0}} \\
& +\left\{a_{k}^{(2)}(2 ; x) \frac{d}{d x}\left[\delta a^{(1)}(x)\right]+\left[(\delta \ell) \frac{d a^{(1)}(x)}{d x}\right] \frac{d a_{k}^{(2)}(2 ; x)}{d x}\right\}_{x=\ell^{0}} \\
& +\left\{\delta T(x) k\left(T^{0}\right) \frac{d a_{k}^{(2)}(1 ; x)}{d x}\right\}_{x=0}+\left\{\delta a^{(1)}(x) \frac{d a_{k}^{(2)}(2 ; x)}{d x}\right\}_{x=0} .
\end{aligned}
$$

The first term on the right side of Equation (136) is now required to represent the indirect-effect term defined in Equation (123), which is accomplished by imposing the following relation:

$$
\left\{\mathbf{A M}^{(2)}(2 \times 2 ; \boldsymbol{\alpha}) \mathbf{A}_{k}^{(2)}(2 ; x)\right\}_{\boldsymbol{\alpha}^{0}}=\left\{\mathbf{Q}_{A}^{(2)}(2 ; \boldsymbol{\alpha})\right\}_{\boldsymbol{\alpha}^{0^{\prime}}}, x \in \Omega_{x}
$$

where

$$
\mathbf{Q}_{A}^{(2)}(2 ; \boldsymbol{\alpha}) \triangleq\left(\begin{array}{c}
q_{A}^{(2)}(1 ; \boldsymbol{\alpha}) \\
q_{A}^{(2)}(2 ; \boldsymbol{\alpha})
\end{array}\right) \triangleq\left(\begin{array}{c}
4 k_{0} \beta[1+\beta T(x)] \delta\left(x-x_{r}\right) \\
Q / k_{0}
\end{array}\right)
$$


The definition domain of the adjoint (matrix-valued) operator $\mathbf{A M}^{(2)}(2 \times 2 ; \boldsymbol{\alpha})$ is now specified by requiring the function $\mathbf{A}_{k}^{(2)}(2 ; x) \triangleq\left[a_{k}^{(2)}(1 ; x), a_{k}^{(2)}(2 ; x)\right]^{\dagger}$ to satisfy adjoint boundary/initial conditions which fulfill the following conditions: (a) they must be independent of unknown values of $\mathbf{V}^{(2)}(2 ; \mathbf{x})$; (b) they must cause all terms containing unknown values of $\mathbf{V}^{(2)}(2 ; \mathbf{x})$ in the expression of $\left\{\left[P^{(2)}\left(\mathbf{A}_{k}^{(2)} ; \mathbf{V}^{(2)} ; \boldsymbol{\alpha}\right)\right]_{\partial \Omega_{x}}\right\}_{\boldsymbol{\alpha}^{0}}$ to vanish. The above requirements are satisfied by imposing the following boundary conditions:

$$
\begin{aligned}
& \left\{\mathbf{B}_{A}^{(2)}\left(\mathbf{A}_{k}^{(2)} ; \boldsymbol{\alpha}\right)\right\}_{\boldsymbol{\alpha}^{0}} \triangleq\left\{\left[b_{A}^{(2)}\left(1 ; \mathbf{A}_{k}^{(2)} ; \boldsymbol{\alpha}\right), \ldots, b_{A}^{(2)}\left(4 ; \mathbf{A}_{k}^{(2)} ; \boldsymbol{\alpha}\right)\right]^{\dagger}\right\}_{\boldsymbol{\alpha}^{0}}=0 ; x \in \partial \Omega \\
& \text { where: } \begin{aligned}
& b_{A}^{(2)}\left(1 ; \mathbf{A}_{k}^{(2)} ; \boldsymbol{\alpha}\right) \triangleq a_{k}^{(2)}(1 ; x=\ell), \\
& b_{A}^{(2)}\left(1 ; \mathbf{A}_{k}^{(2)} ; \boldsymbol{\alpha}\right) \triangleq\left\{d a_{k}^{(2)}(1 ; x) / d x\right\}_{x=0^{\prime}} \\
& b_{A}^{(2)}\left(3 ; \mathbf{A}_{k}^{(2)} ; \boldsymbol{\alpha}\right) \triangleq a_{k}^{(2)}(2 ; x=\ell), \\
& b_{A}^{(2)}\left(1 ; \mathbf{A}_{k}^{(2)} ; \boldsymbol{\alpha}\right) \triangleq\left\{d a_{k}^{(2)}(2 ; x) / d x\right\}_{x=0^{\prime}}
\end{aligned}
\end{aligned}
$$

The system of equations comprising Equations (140) and (142) constitutes the 2ndLASS for the 2nd-level adjoint function $\mathbf{A}_{k}^{(2)}(2 ; x) \triangleq\left[a_{k}^{(2)}(1 ; x), a_{k}^{(2)}(2 ; x)\right]^{\dagger}$.

Using the equations underlying the 2nd-LASS together with Equations (134) and (139) in Equation (136) yields the following expression for the indirect-effect term defined in Equation (123):

$$
\begin{gathered}
\left\{\delta\left[\partial R / \partial k_{0}\right]\right\}_{\text {ind }}=\left\{\int_{0}^{\ell} a_{k}^{(2)}(1 ; x) q_{V}^{(2)}(1 ; \boldsymbol{\alpha} ; \delta \boldsymbol{\alpha}) d x\right\}_{\boldsymbol{\alpha}^{0}}+\left\{\int_{0}^{\ell} a_{k}^{(2)}(2 ; x) q_{V}^{(2)}(2 ; \boldsymbol{\alpha} ; \delta \boldsymbol{\alpha}) d x\right\}_{\boldsymbol{\alpha}^{0}} \\
-(\delta \ell)\left\{k\left(T^{0}\right) \frac{d T(x)}{d x} \frac{d a_{k}^{(2)}(1 ; x)}{d x}+\frac{d a^{(1)}(x)}{d x} \frac{d a_{k}^{(2)}(2 ; x)}{d x}\right\}_{x=\ell^{0}}
\end{gathered}
$$

Replacing in Equation (147) the source terms $q_{V}^{(2)}(1 ; \boldsymbol{\alpha} ; \delta \boldsymbol{\alpha})$ and $q_{V}^{(2)}(2 ; \boldsymbol{\alpha} ; \delta \boldsymbol{\alpha})$ with their expressions provided in Equation (130) and adding the resulting equation to the expression for the direct-effect term provided in Equation (122) yields the following expression for the G-differential, defined in Equation (121):

$$
\begin{aligned}
& \left\{\delta\left[\frac{\partial R}{\partial k_{0}}\right]\right\}_{\alpha^{0}}=\left\{\int_{0}^{\ell} a_{k}^{(2)}(1 ; x)\left[-(\delta Q)+\left(\delta k_{0}\right) \frac{Q}{k_{0}}-(\delta \beta) k_{0} \frac{d}{d x}\left(T(x) \frac{d T(x)}{d x}\right)\right] d x\right\}_{\alpha^{0}} \\
& +\left\{\int_{0}^{\ell} a_{k}^{(2)}(2 ; x)\left[\left(\delta k_{0}\right) 2 \beta \delta\left(x-x_{r}\right)+(\delta \beta) 2 k_{0} \delta\left(x-x_{r}\right)-\left(\delta x_{r}\right) 2 k_{0} \beta \delta^{\prime}\left(x-x_{r}\right)\right] d x\right\}_{\alpha^{0}} \\
& -(\delta \ell)\left\{k\left(T^{0}\right) \frac{d T(x)}{d x} \frac{d a_{k}^{(2)}(1 ; x)}{d x}+\frac{d a^{(1)}(x)}{d x} \frac{d a_{k}^{(2)}(2 ; x)}{d x}\right\}_{x=\ell^{0}} \\
& +(\delta Q)\left\{\frac{1}{k_{0}} \int_{0}^{\ell} a^{(1)}(x) d x\right\}_{\alpha^{0}}-\left(\delta k_{0}\right)\left\{\frac{Q}{k_{0}^{2}} \int_{0}^{\ell} a^{(1)}(x) d x-2 \int_{0}^{\ell}[1+\beta T(x)]^{2} \delta\left(x-x_{r}\right) d x\right\}_{\alpha^{0}} \\
& +(\delta \beta)\left\{4 k_{0}^{\ell} \int_{0}^{\ell}[1+\beta T(x)] T(x) \delta\left(x-x_{r}\right) d x\right\}_{\alpha^{0}} \\
& -\left(\delta x_{r}\right)\left\{2 k_{0}^{\ell_{0}^{0}}[1+\beta T(x)]^{2} \delta^{\prime}\left(x-x_{r}\right) d x\right\}_{\alpha^{0}} .
\end{aligned}
$$


Identifying in Equation (148) the quantities that multiply the various parameter variations yields the following expressions for the respective partial second-order sensitivities:

$$
\begin{gathered}
\frac{\partial^{2} R}{\partial Q \partial k_{0}}=-\int_{0}^{\ell} a_{k}^{(2)}(1 ; x) d x+\frac{1}{k_{0}} \int_{0}^{\ell} a^{(1)}(x) d x, \\
\frac{\partial^{2} R}{\partial k_{0} \partial k_{0}}=\frac{Q}{k_{0}} \int_{0}^{\ell} a_{k}^{(2)}(1 ; x) d x+2 \beta \int_{0}^{\ell} a_{k}^{(2)}(2 ; x) \delta\left(x-x_{r}\right) d x \\
-\frac{Q}{k_{0}^{2}} \int_{0}^{\ell} a^{(1)}(x) d x+2 \int_{0}^{\ell}[1+\beta T(x)]^{2} \delta\left(x-x_{r}\right) d x, \\
\frac{\partial^{2} R}{\partial \beta \partial k_{0}}=-k_{0} \int_{0}^{\ell} a_{k}^{(2)}(1 ; x) \frac{d}{d x}\left[T(x) \frac{d T(x)}{d x}\right] d x+2 k_{0} \int_{0}^{\ell} a_{k}^{(2)}(2 ; x) \delta\left(x-x_{r}\right) d x \\
+4 k_{0} \int_{0}^{\ell}[1+\beta T(x)] T(x) \delta\left(x-x_{r}\right) d x, \int_{0}^{\partial^{2} R}=-2 k_{0} \beta \int_{0}^{\ell} a_{k}^{(2)}(2 ; x) \delta^{\prime}\left(x-x_{r}\right) d x-2 k_{0}[1+\beta T(x)]^{2} \delta^{\prime}\left(x-x_{r}\right) d x \\
\frac{\partial^{2} R}{\partial \ell \partial k_{0}}=-\left\{k\left(T^{0}\right) \frac{d T(x)}{d x} \frac{d a_{k}^{(2)}(1 ; x)}{d x}+\frac{d a^{(1)}(x)}{d x} \frac{d a_{k}^{(2)}(2 ; x)}{d x}\right\}
\end{gathered}
$$

The expressions presented in Equations (149)-(153) are to be evaluated at the nominal values of the model parameters and state functions but the indicator \{\}$_{\alpha^{0}}$ has been omitted for simplicity. As the expressions and values for the functions $T(x)$ and $a^{(1)}(x)$ are already available, the expressions provided in Equations (149)-(153) can be evaluated by using quadrature formulas after having determined the 2nd-level adjoint function $\mathbf{A}_{k}^{(2)}(2 ; x) \triangleq\left[a_{k}^{(2)}(1 ; x), a_{k}^{(2)}(2 ; x)\right]^{\dagger}$ by solving the 2nd-LASS. Solving Equation (140) subject to the boundary conditions provided in Equation (142) yields the following expressions:

$$
\begin{gathered}
a_{k}^{(2)}(1 ; x)=4 \beta\left[\left(x-x_{r}\right) H\left(x-x_{r}\right)-\ell+x_{r}\right] \\
a_{k}^{(2)}(2 ; x)=Q\left(x^{2}-\ell^{2}\right) / 2 k_{0} .
\end{gathered}
$$

Inserting the expressions for $T(x), a^{(1)}(x)$ and $\mathbf{A}_{k}^{(2)}(2 ; x) \triangleq\left[a_{k}^{(2)}(1 ; x), a_{k}^{(2)}(2 ; x)\right]^{\dagger}$ in Equations (149)-(153) and performing the operations indicated on the right sides of these equations yields the following closed-form expressions for the respective second-order sensitivities:

$$
\begin{gathered}
\frac{\partial^{2} R}{\partial Q \partial k_{0}}=\beta\left(\ell^{2}-x_{r}^{2}\right), \\
\frac{\partial^{2} R}{\partial k_{0} \partial k_{0}}=2, \\
\frac{\partial^{2} R}{\partial \beta \partial k_{0}}=Q\left(\ell^{2}-x_{r}^{2}\right) \\
\frac{\partial^{2} R}{\partial k_{0} \partial x_{r}}=-2 \beta Q x_{r}, \\
\frac{\partial^{2} R}{\partial \ell \partial k_{0}}=2 \beta Q \ell .
\end{gathered}
$$


It is important to note that the symmetry relationship $\partial^{2} R / \partial k_{0} \partial Q=\partial^{2} R / \partial Q \partial k_{0}$ provides the following equality between the expressions provided in Equations (112) and (149):

$$
\frac{\partial^{2} R}{\partial k_{0} \partial Q}=2 \beta \int_{0}^{\ell} a_{Q}^{(2)}(x) \delta\left(x-x_{r}\right) d x=\frac{1}{k_{0}} \int_{0}^{\ell} a^{(1)}(x) d x-\int_{0}^{\ell} a_{k}^{(2)}(1 ; x) d x=\frac{\partial^{2} R}{\partial Q \partial k_{0}},
$$

The identity provided in Equation (161) enables an intrinsic mutual verification of the accuracy of the numerical computation of the respective adjoint functions.

As this paradigm illustrative heat conduction model comprises five uncertain model parameters, there will be a total of 25 second-order sensitivities of the response $R(T ; \boldsymbol{\alpha})$ with respect to the model parameters. Of these 25 second-order sensitivities, 15 will be distinct, while the others are not because of the intrinsic symmetries of the 2nd-order mixed derivatives. Of the 25 second-order sensitivities, 10 have been obtained in Section 3.2. As the computation of the remaining 15 second-order sensitivities of the response $R(T ; \boldsymbol{\alpha})$ with respect to the model parameters, are to be performed by applying the same 4th-CASAM-N principles, as illustrated thus far in Section 3.2, the respective derivations will be omitted for brevity. It is important to note that if all of these computations are performed, the mixed 2nd-order sensitivities of $R(T ; \boldsymbol{\alpha})$ will have been computed twice, due to their symmetries, in the same manner as indicated in Equation (161). These symmetry features enable the intrinsic mutual verification of the accuracy of the numerical computation of all of the 1st-level and 2nd-level adjoint functions involved in the respective computations.

\subsection{Third-Order Response Sensitivities}

As has been shown in Section 3.1, this paradigm illustrative heat conduction model comprises five uncertain model parameters, which means that each model response will have five first-order sensitivities. Applying the principles underlying the determination of all of the 1st-order sensitivities requires a single 1st-level adjoint function. Each of the first-order sensitivities is treated as a response for the computation of the corresponding five second-order sensitivities, which are in turn computed using a single 2nd-level adjoint function. Thus, at most, five second-level adjoint functions will suffice to obtain all of the 25 second-order sensitivities, of which 15 will be distinct. Each of the second-order sensitivities will give rise to five third-order sensitivities, which will be computed using a single 3rd-level adjoint function. This section will illustrate the application of the principles of the 4th-CASAM-N methodology to derive the exact expressions for representative 3rd-order sensitivities corresponding to the 2nd-order sensitivities obtained in Section 3.2. Examining the expressions of the 2nd-order sensitivities obtained in Section 3.2, it becomes apparent that that the 3rd-level adjoint functions, which would be needed to compute the 3rd-order sensitivities, will comprise as few as one component (if the originating 2nd-order sensitivity depends on a single component of the state function or the 1st- and 2nd-level adjoint functions) or as many as four components, if the originating 2nd-order sensitivity depends on all state and adjoint functions, namely $T(x), a^{(1)}(x)$ and $\mathbf{A}_{k}^{(2)}(2 ; x) \triangleq\left[a_{k}^{(2)}(1 ; x), a_{k}^{(2)}(2 ; x)\right]^{\dagger}$.

It is instructive to illustrate the consequence of the symmetry properties of the 2nd-order sensitivities by means of the equality relation indicated in Equation (161) between the two alternative expressions for $\partial^{2} R / \partial k_{0} \partial Q$. Using these two alternative expressions, this section will illustrate the symmetry properties of the 3rd-order sensitivities that arise from the symmetry properties of $\partial^{2} R / \partial k_{0} \partial Q$.

Applying the definition of the G-differential to Equation (149) yields the following expression: 


$$
\begin{aligned}
& \left\{\delta\left[\frac{\partial^{2} R}{\partial k_{0} \partial Q}\right]\right\}_{\alpha^{0}} \triangleq\left\{\frac{d}{d \varepsilon}\left[\frac{1}{k_{0}^{0}+\varepsilon \delta k_{0}} \int_{0}^{\ell^{0}+\varepsilon \delta \ell}\left[a^{(1), 0}(x)+\varepsilon \delta a^{(1)}(x)\right] d x\right]\right\}_{\varepsilon=0} \\
& -\left\{\frac{d}{d \varepsilon} \int_{0}^{\ell^{0}+\varepsilon \delta \ell}\left[a_{k}^{(2), 0}(1 ; x)+\varepsilon \delta a_{k}^{(2)}(1 ; x)\right] d x\right\}_{\varepsilon=0} \\
& \triangleq\left\{\delta\left[\partial^{2} R / \partial Q \partial k_{0}\right]\right\}_{d i r}+\left\{\delta\left[\partial^{2} R / \partial Q \partial k_{0}\right]\right\}_{\text {ind }} .
\end{aligned}
$$

The direct-effect term $\left\{\delta\left[\partial^{2} R / \partial Q \partial k_{0}\right]\right\}_{\text {dir }}$ is defined to depend only on parameter variations and has the following expression:

$$
\begin{aligned}
& \left\{\delta\left[\partial^{2} R / \partial Q \partial k_{0}\right]\right\}_{d i r} \triangleq-\left(\delta k_{0}\right)\left\{\frac{1}{k_{0}^{2}} \int_{0}^{\ell} a^{(1)}(x) d x\right\}_{\alpha^{0}} \\
& +(\delta \ell)\left\{\frac{a^{(1)}(x=\ell)}{k_{0}}-a_{k}^{(2)}(1 ; x=\ell)\right\}_{\alpha^{0}}=-\left(\delta k_{0}\right)\left\{\frac{1}{k_{0}^{2}} \int_{0}^{\ell} a^{(1)}(x) d x\right\}_{\alpha^{0}} .
\end{aligned}
$$

The boundary terms on the right side of Equation (163) vanish in view of the boundary conditions given in Equations (79) and (142). The direct-effect term can be evaluated at this stage.

The indirect-effect term $\left\{\delta\left[\partial^{2} R / \partial Q \partial k_{0}\right]\right\}_{\text {ind }}$ is defined to depend only on the variations $\delta a^{(1)}(x)$ and $\delta a_{k}^{(2)}(1 ; x)$; it has the following expression:

$$
\left\{\delta\left[\partial^{2} R / \partial Q \partial k_{0}\right]\right\}_{\text {ind }} \triangleq\left\{\frac{1}{k_{0}} \int_{0}^{\ell} \delta a^{(1)}(x) d x\right\}_{\alpha^{0}}-\left\{\int_{0}^{\ell} \delta a_{k}^{(2)}(1 ; x) d x\right\}_{\alpha^{0}} .
$$

The variation $\delta a^{(1)}(x)$ is the solution of the 2nd-LVSS defined in Equations (101)-(103). The variation $\delta a_{k}^{(2)}(1 ; x)$ is the solution of the system of equations obtained by taking the G-differential of the 2nd-LASS defined in Equations (140) and (142), which yields:

$$
\begin{gathered}
\left\{\frac{d^{2}\left[\delta a_{k}^{(2)}(1 ; x)\right]}{d x^{2}}\right\}_{\alpha^{0}}=\left\{4(\delta \beta) \delta\left(x-x_{r}\right)-4\left(\delta x_{r}\right) \beta \delta^{\prime}\left(x-x_{r}\right)\right\}_{\alpha^{0}}, 0<x<\ell^{0} ; \\
\delta a_{k}^{(2)}(1 ; x)+(\delta \ell)\left\{\frac{d a_{k}^{(2)}(1 ; x)}{d x}\right\}_{\alpha^{0}}=0 \text {, at } x=\ell^{0} ; \\
\frac{d\left[\delta a_{k}^{(2)}(1 ; x)\right]}{d x}=0, \text { at } x=0 .
\end{gathered}
$$

The system comprising Equations (101)-(103) and (165)-(167) constitutes the 3rd-level variational sensitivity system (3rd-LVSS) for the functions $\delta a^{(1)}(x)$ and $\delta a_{k}^{(2)}(1 ; x)$. Rather than solving this 3rd-LVSS repeatedly for the various parameter variations, it is computationally considerably more advantageous to recast the indirect-effect term defined in Equation (164) in terms of a third-level adjoint function, which will be the solution of a 3rd-level adjoint sensitivity system (3rd-LASS) to be constructed by applying the principles of the 4th-CASAM-N methodology. As the indirect-effect term depends on two variational functions, $\delta a^{(1)}(x)$ and $\delta a_{k}^{(2)}(1 ; x)$, it follows that the 3rd-level adjoint function will be a two-component vector of the form $\mathbf{A}_{k}^{(3)}(2 ; x) \triangleq\left[a_{k}^{(3)}(1 ; x), a_{k}^{(3)}(2 ; x)\right]^{\dagger} \equiv$ $\left[a_{k}^{(3)}(1 ; x), a_{k}^{(3)}(2 ; x) ; a_{k}^{(3)}(3 ; x) \equiv 0, a_{k}^{(3)}(4 ; x) \equiv 0\right]^{\dagger}$, rather than a four-component vector, as would have been the case if the indirect-effect term had depended on a four- 
component variational vector comprising the functions $\delta T(x), \delta a^{(1)}(x)$ and $\delta \mathbf{A}_{k}^{(2)}(2 ; x) \triangleq$ $\left[\delta a_{k}^{(2)}(1 ; x), \delta a_{k}^{(2)}(2 ; x)\right]^{\dagger}$.

Thus, the 3rd-LASS, which corresponds to the 3rd-LVSS comprising Equations (101)-(103) and (165)-(167), can be constructed using the inner product provided in Equation (133) and following the same conceptual steps as presented in Section 3.2.2. The main conceptual steps for constructing the requisite 3rd-LASS are as follows:

1. Using Equation (133), construct the inner product of a yet undefined vector $\mathbf{A}_{k}^{(3)}(2 ; x) \triangleq$ $\left[a_{k}^{(3)}(1 ; x), a_{k}^{(3)}(2 ; x)\right]^{\dagger}$ with Equations (101) and (165) to obtain the following relation:

$\left\{\int_{0}^{\ell} a_{k}^{(3)}(1 ; x) \frac{d^{2}\left[\delta a^{(1)}(x)\right]}{d x^{2}} d x\right\}_{\alpha^{0}}+\left\{\int_{0}^{\ell} a_{k}^{(3)}(2 ; x) \frac{d^{2}\left[\delta a_{k}^{(2)}(1 ; x)\right]}{d x^{2}} d x\right\}_{\alpha^{0}}$

$=\left(\delta k_{0}\right)\left\{2 \beta \int_{0}^{\ell} a_{k}^{(3)}(1 ; x) \delta\left(x-x_{r}\right) d x\right\}_{\alpha^{0}}+(\delta \beta)\left\{2 k_{0} \int_{0}^{\ell} a_{k}^{(3)}(1 ; x) \delta\left(x-x_{r}\right) d x\right\}_{\boldsymbol{\alpha}^{0}}$

$-\left(\delta x_{r}\right)\left\{2 k_{0} \beta \int_{0}^{\ell} a_{k}^{(3)}(1 ; x) \delta^{\prime}\left(x-x_{r}\right) d x\right\}_{\alpha^{0}}+(\delta \beta)\left\{4 \int_{0}^{\ell} a_{k}^{(3)}(2 ; x) \delta\left(x-x_{r}\right) d x\right\}_{\alpha^{0}}$

$-\left(\delta x_{r}\right)\left\{4 \beta \int_{0}^{\ell} a_{k}^{(3)}(2 ; x) \delta^{\prime}\left(x-x_{r}\right) d x\right\}_{\alpha^{0}}, 0<x<\ell^{0}$.

2. Integrate the left side of Equation (168) twice by parts to obtain the following relation:

$\left\{\int_{0}^{\ell} a_{k}^{(3)}(1 ; x) \frac{d^{2}\left[\delta a^{(1)}(x)\right]}{d x^{2}} d x\right\}_{\alpha^{0}}+\left\{\int_{0}^{\ell} a_{k}^{(3)}(2 ; x) \frac{d^{2}\left[\delta a_{k}^{(2)}(1 ; x)\right]}{d x^{2}} d x\right\}_{\alpha^{0}}$

$=\left\{\int_{0}^{\ell} \delta a^{(1)}(x) \frac{d^{2}\left[a_{k}^{(3)}(1 ; x)\right]}{d x^{2}} d x\right\}_{\alpha^{0}}+\left\{\int_{0}^{\ell} \delta a_{k}^{(2)}(1 ; x) \frac{d^{2}\left[a_{k}^{(3)}(2 ; x)\right]}{d x^{2}} d x\right\}_{\alpha^{0}}$

$+\left\{a_{k}^{(3)}(1 ; x) \frac{d}{d x}\left[\delta a^{(1)}(x)\right]-\delta a^{(1)}(x) \frac{d}{d x}\left[a_{k}^{(3)}(1 ; x)\right]\right\}_{x=0}^{x=\ell}$

$+\left\{a_{k}^{(3)}(2 ; x) \frac{d}{d x}\left[\delta a_{k}^{(2)}(1 ; x)\right]-\delta a_{k}^{(2)}(1 ; x) \frac{d}{d x}\left[a_{k}^{(3)}(2 ; x)\right]\right\}_{x=0}^{x=\ell}$.

3. Inserting into Equation (169) the boundary conditions provided in Equations (102), (103), (166) and (167) makes it possible to recast Equation (169) into the following form:

$$
\begin{aligned}
& \left\{\int_{0}^{\ell} \delta a^{(1)}(x) \frac{d^{2}\left[a_{k}^{(3)}(1 ; x)\right]}{d x^{2}} d x\right\}_{\alpha^{0}}+\left\{\int_{0}^{\ell} \delta a_{k}^{(2)}(1 ; x) \frac{d^{2}\left[a_{k}^{(3)}(2 ; x)\right]}{d x^{2}} d x\right\}_{\alpha^{0}} \\
& =\left\{\int_{0}^{\ell} a_{k}^{(3)}(1 ; x) \frac{d^{2}\left[\delta a^{(1)}(x)\right]}{d x^{2}} d x\right\}_{\alpha^{0}}+\left\{\int_{0}^{\ell} a_{k}^{(3)}(2 ; x) \frac{d^{2}\left[\delta a_{k}^{(2)}(1 ; x)\right]}{d x^{2}} d x\right\}_{\alpha^{0}} \\
& -\left\{a_{k}^{(3)}(1 ; x) \frac{d}{d x}\left[\delta a^{(1)}(x)\right]+(\delta \ell) \frac{d a^{(1)}(x)}{d x} \frac{d}{d x}\left[a_{k}^{(3)}(1 ; x)\right]\right\}_{x=\ell} \\
& -\left\{\delta a^{(1)}(x) \frac{d}{d x}\left[a_{k}^{(3)}(1 ; x)\right]\right\}_{x=0}-\left\{\delta a_{k}^{(2)}(1 ; x) \frac{d}{d x}\left[a_{k}^{(3)}(2 ; x)\right]\right\}_{x=0} \\
& -\left\{a_{k}^{(3)}(2 ; x) \frac{d}{d x}\left[\delta a_{k}^{(2)}(1 ; x)\right]+(\delta \ell) \frac{d a_{k}^{(2)}(1 ; x)}{d x} \frac{d}{d x}\left[a_{k}^{(3)}(2 ; x)\right]\right\}_{x=\ell} .
\end{aligned}
$$


4. The left side of Equation (170) is required to represent the indirect-effect term defined in Equation (164) and the unknown values of the functions are eliminated from the right side of Equation (170) by imposing the following relations:

$$
\begin{gathered}
\left\{\frac{d^{2}\left[a_{k}^{(3)}(1 ; x)\right]}{d x^{2}}\right\}_{\alpha^{0}}=\left\{\frac{1}{k_{0}}\right\}_{\alpha^{0}}, 0<x<\ell^{0} ; \\
\frac{d}{d x}\left[a_{k}^{(3)}(1 ; x)\right]=0, \text { at } x=0 ; \\
\frac{a_{k}^{(3)}(1 ; x)=0, \text { at } x=\ell^{0}}{\frac{d^{2}\left[a_{k}^{(3)}(2 ; x)\right]}{d x^{2}}=-1,0<x<\ell^{0}} \\
\frac{d}{d x}\left[a_{k}^{(3)}(2 ; x)\right]=0, \text { at } x=0 ; \\
a_{k}^{(3)}(2 ; x)=0, \text { at } x=\ell^{0}
\end{gathered}
$$

5. The relations indicated in Equations (171)-(176) constitute the 3rd-LASS to be satisfied by the 3rd-level adjoint function $\mathbf{A}_{k}^{(3)}(2 ; x) \triangleq\left[a_{k}^{(3)}(1 ; x), a_{k}^{(3)}(2 ; x)\right]^{\dagger}$. Inserting the equations underlying this 3rd-LASS into Equation (170) reduces the latter to the following form:

$$
\begin{aligned}
& \left\{\delta\left[\partial^{2} R / \partial Q \partial k_{0}\right]\right\}_{\text {ind }}=\left\{\int_{0}^{\ell} a_{k}^{(3)}(1 ; x) \frac{d^{2}\left[\delta a^{(1)}(x)\right]}{d x^{2}} d x\right\}_{\alpha^{0}} \\
& +\left\{\int_{0}^{\ell} a_{k}^{(3)}(2 ; x) \frac{d^{2}\left[\delta a_{k}^{(2)}(1 ; x)\right]}{d x^{2}} d x\right\} \\
& -(\delta \ell)\left\{\frac{d a^{(1)}(x)}{d x} \frac{d}{d x}\left[a_{k}^{(3)}(1 ; x)\right]+\frac{d a_{k}^{(2)}(1 ; x)}{d x} \frac{d}{d x}\left[a_{k}^{(3)}(2 ; x)\right]\right\}_{x=\ell} .
\end{aligned}
$$

6. The two variational terms that appear in the first two terms on the right side of Equation (177) are now replaced by their equivalent expressions provided on the left sides of Equations (101) and (165), respectively, to obtain the following relation:

$$
\begin{aligned}
& \left\{\delta\left[\partial^{2} R / \partial Q \partial k_{0}\right]\right\}_{\text {ind }}=\left(\delta k_{0}\right)\left\{2 \beta \int_{0}^{\ell} a_{k}^{(3)}(1 ; x) \delta\left(x-x_{r}\right) d x\right\}_{\alpha^{0}} \\
& +(\delta \beta)\left\{2 k_{0} \int_{0}^{\ell} a_{k}^{(3)}(1 ; x) \delta\left(x-x_{r}\right) d x\right\}_{\alpha^{0}}-\left(\delta x_{r}\right)\left\{2 k_{0} \beta \int_{0}^{\ell} a_{k}^{(3)}(1 ; x) \delta^{\prime}\left(x-x_{r}\right) d x\right\}_{\alpha^{0}} \\
& +(\delta \beta)\left\{4 \int_{0}^{\ell} a_{k}^{(3)}(2 ; x) \delta\left(x-x_{r}\right) d x\right\}-\left(\delta x_{r}\right)\left\{4 \beta \int_{0}^{\ell} a_{k}^{(3)}(2 ; x) \delta^{\prime}\left(x-x_{r}\right) d x\right\}_{\alpha^{0}} \\
& -(\delta \ell)\left\{\frac{d a^{(1)}(x)}{d x} \frac{d\left[a_{k}^{(3)}(1 ; x)\right]}{d x}+\frac{d a_{k}^{(2)}(1 ; x)}{d x} \frac{d\left[a_{k}^{(3)}(2 ; x)\right]}{d x}\right\}_{x=\ell} .
\end{aligned}
$$

Presently inserting into Equation (162) the expression of the indirect-effect term obtained in Equation (178), together with the expression of the direct-effect term obtained in Equation (163), yields the following expression for the G-variation $\left\{\delta\left[\partial^{2} R / \partial k_{0} \partial Q\right]\right\} \alpha_{\alpha^{0}}$ :

$$
\begin{aligned}
& \left\{\delta\left[\frac{\partial^{2} R}{\partial k_{0} \partial Q}\right]\right\}_{\alpha^{0}} \triangleq\left\{\frac{\partial^{3} R}{\partial k_{0} \partial^{2} Q}\right\}_{\alpha^{0}} \delta Q+\left\{\frac{\partial^{3} R}{\partial^{2} k_{0} \partial Q}\right\}_{\alpha^{0}} \delta k_{0}+\left\{\frac{\partial^{3} R}{\partial \beta \partial k_{0} \partial Q}\right\}_{\alpha^{0}} \delta \beta \\
& +\left\{\frac{\partial^{3} R}{\partial x_{r} \partial k_{0} \partial Q}\right\}_{\alpha^{0}} \delta x_{r}+\left\{\frac{\partial^{3} R}{\partial \ell \partial k_{0} \partial Q}\right\}_{\alpha^{0}} \delta \ell,
\end{aligned}
$$


where the respective 3rd-order response sensitivities have the following expressions:

$$
\begin{gathered}
\frac{\partial^{3} R}{\partial k_{0} \partial^{2} Q} \equiv 0, \\
\frac{\partial^{3} R}{\partial^{2} k_{0} \partial Q}=2 \beta \int_{0}^{\ell} a_{k}^{(3)}(1 ; x) \delta\left(x-x_{r}\right) d x-\frac{1}{k_{0}^{2}} \int_{0}^{\ell} a^{(1)}(x) d x, \\
\frac{\partial^{3} R}{\partial \beta \partial k_{0} \partial Q}=2 k_{0} \int_{0}^{\ell} a_{k}^{(3)}(1 ; x) \delta\left(x-x_{r}\right) d x+4 \int_{0}^{\ell} a_{k}^{(3)}(2 ; x) \delta\left(x-x_{r}\right) d x, \\
\frac{\partial^{3} R}{\partial x_{r} \partial k_{0} \partial Q}=-2 k_{0} \beta \int_{0}^{\ell} a_{k}^{(3)}(1 ; x) \delta^{\prime}\left(x-x_{r}\right) d x-4 \beta \int_{0}^{\ell} a_{k}^{(3)}(2 ; x) \delta^{\prime}\left(x-x_{r}\right) d x, \\
\frac{\partial^{3} R}{\partial \ell \partial k_{0} \partial Q}=-\left\{\frac{d a^{(1)}(x)}{d x} \frac{d\left[a_{k}^{(3)}(1 ; x)\right]}{d x}+\frac{d a_{k}^{(2)}(1 ; x)}{d x} \frac{d\left[a_{k}^{(3)}(2 ; x)\right]}{d x}\right\} .
\end{gathered} .
$$

Solving the 3rd-LASS analytically yields the following expressions for the components of the 3rd-level adjoint function $\mathbf{A}_{k}^{(3)}(2 ; x) \triangleq\left[a_{k}^{(3)}(1 ; x), a_{k}^{(3)}(2 ; x)\right]^{\dagger}$ :

$$
\begin{aligned}
& a_{k}^{(3)}(1 ; x)=\frac{x^{2}-\ell^{2}}{2 k_{0}}, \\
& a_{k}^{(3)}(2 ; x)=\frac{\ell^{2}-x^{2}}{2} .
\end{aligned}
$$

Using the expressions obtained in Equations (185) and (186) together with the requisite expressions for the various functions that have been already obtained in Equations (181)-(184) yields the following closed-form analytical expressions for the respective 3rd-order sensitivities:

$$
\begin{gathered}
\frac{\partial^{3} R}{\partial^{2} k_{0} \partial Q}=0, \\
\frac{\partial^{3} R}{\partial \beta \partial k_{0} \partial Q}=\ell^{2}-x_{r}^{2}, \\
\frac{\partial^{3} R}{\partial x_{r} \partial k_{0} \partial Q}=-2 \beta x_{r}, \\
\frac{\partial^{3} R}{\partial \ell \partial k_{0} \partial Q}=2 \beta \ell .
\end{gathered}
$$

On the other hand, the 3rd-order sensitivities stemming from $\partial^{2} R / \partial k_{0} \partial Q$ can be obtained by using the alternative expression for $\partial^{2} R / \partial k_{0} \partial Q$ provided in Equation (112). Applying the definition of the G-differential to Equation (112) yields the following expression:

$$
\begin{aligned}
& \left\{\delta\left[\frac{\partial^{2} R}{\partial k_{0} \partial Q}\right]\right\}_{\alpha^{0}} \triangleq\left\{\frac{d}{d \varepsilon}\left[2\left(\beta^{0}+\varepsilon \delta \beta\right) \int_{0}^{\ell^{0}+\varepsilon \delta \ell}\left(a_{Q}^{(2), 0}+\varepsilon \delta a_{Q}^{(2)}\right) \delta\left(x-x_{r}^{0}-\varepsilon \delta x_{r}\right) d x\right]\right\}_{\varepsilon=0} \\
& \triangleq\left\{\delta\left[\partial^{2} R / \partial Q \partial k_{0}\right]\right\}_{d i r}+\left\{\delta\left[\partial^{2} R / \partial Q \partial k_{0}\right]\right\}_{\text {ind }} .
\end{aligned}
$$

The direct-effect term $\left\{\delta\left[\partial^{2} R / \partial Q \partial k_{0}\right]\right\}_{\text {dir }}$ is defined to depend only on parameter variations and has the following expression: 


$$
\begin{aligned}
& \left\{\delta\left[\partial^{2} R / \partial Q \partial k_{0}\right]\right\}_{d i r} \triangleq(\delta \beta)\left\{2 \int_{0}^{\ell} a_{Q}^{(2)}(x) \delta\left(x-x_{r}^{0}\right) d x\right\}_{\alpha^{0}} \\
& -\left(\delta x_{r}\right)\left\{2 \beta \int_{0}^{\ell} a_{Q}^{(2)}(x) \delta^{\prime}\left(x-x_{r}^{0}\right) d x\right\}_{\alpha^{0}} .
\end{aligned}
$$

The direct-effect term can be evaluated at this stage. The indirect-effect term $\left\{\delta\left[\partial^{2} R / \partial Q \partial k_{0}\right]\right\}_{\text {ind }}$ is defined to depend only on the variation $\delta a_{Q}^{(2)}(x)$ and has the following expression:

$$
\left\{\delta\left[\partial^{2} R / \partial Q \partial k_{0}\right]\right\}_{\text {ind }} \triangleq\left\{2 \beta \int_{0}^{\ell} \delta a_{Q}^{(2)}(x) \delta\left(x-x_{r}\right) d x\right\}_{\alpha^{0}} .
$$

The variational function $\delta a_{O}^{(2)}(x)$ is the solution of the system obtained by taking the G-differential of the 2nd-LASS defined by Equations (107)-(109), and which has the following expression:

$$
\begin{gathered}
\frac{d^{2}\left[\delta a_{Q}^{(2)}(x)\right]}{d x^{2}}=0, \quad 0<x<\ell^{0} ; \\
\delta a_{Q}^{(2)}(x)+(\delta \ell)\left\{\frac{d a_{Q}^{(2)}(x)}{d x}\right\}_{\alpha^{0}}=0, \text { at } x=\ell^{0} ; \\
\frac{d\left[\delta a_{Q}^{(2)}(x)\right]}{d x}=0, \text { at } x=0 .
\end{gathered}
$$

The system comprising Equations (194)-(196) represents the 3rd-level variational sensitivity system (3rd-LVSS) for the function $\delta a_{Q}^{(2)}(x)$. Its solution, $\delta a_{Q}^{(2)}(x)$, can be used to determine the indirect-effect term $\left\{\delta\left[\partial^{2} R / \partial Q \partial k_{0}\right]\right\}_{\text {ind }}$ defined in Equation (193). In this extreme case, the 3rd-LVSS for the function $\delta a_{Q}^{(2)}(x)$ depends on a single parameter variation so it would need to be solved only once to determine $\delta a_{O}^{(2)}(x)$. In this extreme case, constructing a 3rd-level adjoint sensitivity system (3rd-LASS) corresponding to the 3rd-LVSS for the function $\delta a_{O}^{(2)}(x)$ would not improve the computational efficiency, as solving the corresponding 3rd-LASS would be comparable to solving the 3rd-LVSS once. Consequently, in this extreme case, the 3rd-LVSS is solved directly to obtain the following expression for $\delta a_{Q}^{(2)}(x)$ :

$$
\delta a_{Q}^{(2)}(x)=\ell(\delta \ell)
$$

Using the result obtained in Equation (197) in Equation (193) yields:

$$
\left\{\delta\left[\partial^{2} R / \partial Q \partial k_{0}\right]\right\}_{\text {ind }}=(\delta \ell)\{2 \beta \ell\}_{\alpha^{0}} .
$$

Using the result obtained in Equation (116) for $a_{Q}^{(2)}(x)$ in Equation (192) yields the following result:

$$
\left\{\delta\left[\partial^{2} R / \partial Q \partial k_{0}\right]\right\}_{d i r} \triangleq(\delta \beta)\left\{\left(\ell^{2}-x_{r}^{2}\right)\right\}_{\alpha^{0}}-2\left(\delta x_{r}\right)\left\{\beta x_{r}\right\}_{\boldsymbol{\alpha}^{0}} .
$$

Adding the results obtained in Equations (198) and (199), and identifying the expressions that multiply the respective parameter variations, yields the following results for the corresponding 3rd-order sensitivities: 


$$
\begin{gathered}
\frac{\partial^{3} R}{\partial k_{0} \partial^{2} Q}=\frac{\partial^{3} R}{\partial^{2} k_{0} \partial Q} \equiv 0, \\
\frac{\partial^{3} R}{\partial \beta \partial k_{0} \partial Q}=\left(\ell^{2}-x_{r}^{2}\right), \\
\frac{\partial^{3} R}{\partial x_{r} \partial k_{0} \partial Q}=-2 \beta x_{r}, \\
\frac{\partial^{3} R}{\partial \ell \partial k_{0} \partial Q}=2 \beta \ell .
\end{gathered}
$$

As has been illustrated in this section by using the two alternative expressions of the 2nd-order sensitivity $\partial^{2} R / \partial Q \partial k_{0}$, the symmetry properties of the 2 nd-order sensitivities enable their selective computation, as well as the verification of the accuracy of the various first- and second-level adjoint functions, when computed numerically, as would be the case in practice.

\subsection{Fourth-Order Response Sensitivities}

The fourth-order sensitivities are determined by considering each of the third-order sensitivities as a "model response" and consequently determining the corresponding G-differential. The concepts underlying the computation of the 4th-order sensitivities can be illustrated by using the expression of the 3rd-order sensitivity $\partial^{3} R / \partial \beta \partial k_{0} \partial Q$, as the requisite algebraic operations that would be required to determine the 4 th-order sensitivities stemming from $\partial^{3} R / \partial \beta \partial k_{0} \partial Q$ are sufficiently transparent to avoid obscuring the underlaying conceptual steps involved in the application of the 4th-CASAM-N. Applying the definition of the G-differential to Equation (182) yields the following relation:

$$
\begin{aligned}
& \left\{\delta\left[\frac{\partial^{3} R}{\partial \beta \partial k_{0} \partial Q}\right]\right\}_{\alpha^{0}} \\
& \triangleq\left\{\frac{d}{d \varepsilon}\left[2\left(k_{0}^{0}+\varepsilon \delta k_{0}\right) \int_{0}^{\ell^{0}+\varepsilon \delta \ell}\left[a_{k}^{(3), 0}(1 ; x)+\varepsilon \delta a_{k}^{(3)}(1 ; x)\right] \delta\left(x-x_{r}^{0}-\varepsilon \delta x_{r}\right) d x\right]\right\}_{\varepsilon=0} \\
& +\left\{\frac{d}{d \varepsilon}\left[4 \int_{0}^{\ell^{0}+\varepsilon \delta \ell}\left[a_{k}^{(3), 0}(2 ; x)+\varepsilon \delta a_{k}^{(3)}(2 ; x)\right] \delta\left(x-x_{r}^{0}-\varepsilon \delta x_{r}\right) d x\right]\right\}_{\varepsilon=0} \\
& \triangleq\left\{\delta\left[\partial^{3} R / \partial \beta \partial k_{0} \partial Q\right]\right\}_{d i r}+\left\{\delta\left[\partial^{3} R / \partial \beta \partial k_{0} \partial Q\right]\right\}_{\text {ind }} .
\end{aligned}
$$

The direct-effect term $\left\{\delta\left[\partial^{3} R / \partial \beta \partial k_{0} \partial Q\right]\right\}_{\text {dir }}$ is defined to depend only on parameter variations and has the following expression:

$$
\begin{aligned}
& \left\{\delta\left[\partial^{3} R / \partial \beta \partial k_{0} \partial Q\right]\right\}_{d i r} \triangleq\left(\delta k_{0}\right)\left\{2 \int_{0}^{\ell} a_{k}^{(3)}(1 ; x) \delta\left(x-x_{r}\right) d x\right\}_{\alpha^{0}} \\
& -\left(\delta x_{r}\right)\left\{\int_{0}^{\ell}\left[2 k_{0} a_{k}^{(3)}(1 ; x)+4 a_{k}^{(3)}(2 ; x)\right] \delta^{\prime}\left(x-x_{r}\right) d x\right\}_{\alpha^{0}} .
\end{aligned}
$$

The indirect-effect term $\left\{\delta\left[\partial^{3} R / \partial \beta \partial k_{0} \partial Q\right]\right\}_{\text {ind }}$ is defined to depend only on the variations $\delta a_{k}^{(3)}(1 ; x)$ and $\delta a_{k}^{(3)}(2 ; x)$; it has the following expression:

$$
\begin{aligned}
& \left\{\delta\left[\partial^{3} R / \partial \beta \partial k_{0} \partial Q\right]\right\}_{\text {ind }} \\
& \triangleq\left\{2 k_{0} \int_{0}^{\ell} \delta a_{k}^{(3)}(1 ; x) \delta\left(x-x_{r}\right) d x\right\}_{\alpha^{0}}+\left\{4 \int_{0}^{\ell} \delta a_{k}^{(3)}(2 ; x) \delta\left(x-x_{r}\right) d x\right\}_{\alpha^{0}} .
\end{aligned}
$$


The variations $\delta a_{k}^{(3)}(1 ; x)$ and $\delta a_{k}^{(3)}(2 ; x)$ are the solutions of the system of equations obtained by taking the G-differential of the 3rd-LASS defined in Equations (142) and (171)-(176), which yields:

$$
\begin{gathered}
\left\{\frac{d^{2}\left[\delta a_{k}^{(3)}(1 ; x)\right]}{d x^{2}}\right\}_{\alpha^{0}}=-\left(\delta k_{0}\right)\left\{\frac{1}{k_{0}^{2}}\right\}_{\alpha^{0}}, 0<x<\ell^{0} ; \\
\frac{d}{d x}\left[\delta a_{k}^{(3)}(1 ; x)\right]=0, \text { at } x=0 ; \\
\delta a_{k}^{(3)}(1 ; x)+(\delta \ell)\left\{\frac{d a_{k}^{(3)}(1 ; x)}{d x}\right\}_{\alpha^{0}}=0, \text { at } x=\ell^{0} ; \\
\left\{\frac{d^{2}\left[\delta a_{k}^{(3)}(2 ; x)\right]}{\left.d x^{2}\right\}}=0,0<x<\ell^{0} ;\right. \\
\frac{d}{d x}\left[\delta a_{k}^{(3)}(2 ; x)\right]=0, \text { at } x=0 ; \\
\delta a_{k}^{(3)}(2 ; x)+(\delta \ell)\left\{\frac{d a_{k}^{(3)}(2 ; x)}{d x}\right\}=0, \text { at } x=\ell^{0} .
\end{gathered}
$$

The system comprising Equations (207)-(212) constitutes the 4th-level variational sensitivity system (4th-LVSS) for the functions $\delta a_{k}^{(3)}(1 ; x)$ and $\delta a_{k}^{(3)}(2 ; x)$. Rather than solving this 4 th-LVSS repeatedly for the various parameter variations, it is computationally considerably more advantageous to recast the indirect-effect term defined in Equation (206) in terms of a fourth-level adjoint function, which will be the solution of a 4th-level adjoint sensitivity system (4th-LASS) to be constructed by applying the principles of the 4th-CASAM-N methodology. As the indirect-effect term depends on two variational functions, $\delta a_{k}^{(3)}(1 ; x)$ and $\delta a_{k}^{(3)}(2 ; x)$, it follows that the 4th-level adjoint function will be a two-component vector of the form $\mathbf{A}_{k}^{(4)}(2 ; x) \triangleq\left[a_{k}^{(4)}(1 ; x), a_{k}^{(4)}(2 ; x)\right]^{\dagger}$. The 4th-LASS, which corresponds to the 4th-LVSS comprising Equations (207)-(212), will be constructed by using the inner product provided in Equation (133) and following the same conceptual steps as presented in Section 3.2.2. The main conceptual steps for constructing the requisite 4th-LASS are as follows:

1. Using Equation (133), construct the inner product of a yet undefined vector $\mathbf{A}_{k}^{(4)}(2 ; x) \triangleq$ $\left[a_{k}^{(4)}(1 ; x), a_{k}^{(4)}(2 ; x)\right]^{\dagger}$ with Equations (207) and (210) to obtain the following relation:

$$
\begin{aligned}
& \left\{\int_{0}^{\ell} a_{k}^{(4)}(1 ; x) \frac{d^{2}\left[\delta a_{k}^{(3)}(1 ; x)\right]}{d x^{2}} d x\right\}_{\alpha^{0}}+\left\{\int_{0}^{\ell} a_{k}^{(4)}(2 ; x) \frac{d^{2}\left[\delta a_{k}^{(3)}(2 ; x)\right]}{d x^{2}} d x\right\}_{\alpha^{0}} \\
& =-\left(\delta k_{0}\right)\left\{\frac{1}{k_{0}^{2}} \int_{0}^{\ell} a_{k}^{(4)}(1 ; x) d x\right\}_{\alpha^{0}} .
\end{aligned}
$$


2. Integrate the left side of Equation (213) twice by parts to obtain the following relation:

$$
\begin{aligned}
& \left\{\int_{0}^{\ell} a_{k}^{(4)}(1 ; x) \frac{d^{2}\left[\delta a_{k}^{(3)}(1 ; x)\right]}{d x^{2}} d x\right\}_{\alpha^{0}}+\left\{\int_{0}^{\ell} a_{k}^{(4)}(2 ; x) \frac{d^{2}\left[\delta a_{k}^{(3)}(2 ; x)\right]}{d x^{2}} d x\right\}_{\alpha^{0}} \\
& =\left\{\int_{0}^{\ell} \delta a_{k}^{(3)}(1 ; x) \frac{d^{2}\left[a_{k}^{(4)}(1 ; x)\right]}{d x^{2}} d x\right\}_{\alpha^{0}}+\left\{\int_{0}^{\ell} \delta a_{k}^{(3)}(2 ; x) \frac{d^{2}\left[a_{k}^{(4)}(2 ; x)\right]}{d x^{2}} d x\right\}_{\alpha^{0}} \\
& +\left\{a_{k}^{(4)}(1 ; x) \frac{d}{d x}\left[\delta a_{k}^{(3)}(1 ; x)\right]-\delta a_{k}^{(3)}(1 ; x) \frac{d}{d x}\left[a_{k}^{(4)}(1 ; x)\right]\right\}_{x=0}^{x=\ell} \\
& +\left\{a_{k}^{(4)}(2 ; x) \frac{d}{d x}\left[\delta a_{k}^{(3)}(2 ; x)\right]-\delta a_{k}^{(3)}(2 ; x) \frac{d}{d x}\left[a_{k}^{(4)}(2 ; x)\right]\right\}_{x=0}^{x=\ell} .
\end{aligned}
$$

3. Inserting into Equation (214) the boundary conditions provided in Equations (208), (209), (211) and (212) makes it possible to recast Equation (214) into the following form:

$$
\begin{aligned}
& \left\{\int_{0}^{\ell} \delta a_{k}^{(3)}(1 ; x) \frac{d^{2}\left[a_{k}^{(4)}(1 ; x)\right]}{d x^{2}} d x\right\}_{\alpha^{0}}+\left\{\int_{0}^{\ell} \delta a_{k}^{(3)}(2 ; x) \frac{d^{2}\left[a_{k}^{(4)}(2 ; x)\right]}{d x^{2}} d x\right\}_{\alpha^{0}} \\
& =\left\{\int_{0}^{\ell} a_{k}^{(4)}(1 ; x) \frac{d^{2}\left[\delta a_{k}^{(3)}(1 ; x)\right]}{d x^{2}} d x\right\}_{\alpha^{0}}+\left\{\int_{0}^{\ell} a_{k}^{(4)}(2 ; x) \frac{d^{2}\left[\delta a_{k}^{(3)}(2 ; x)\right]}{d x^{2}} d x\right\}_{\alpha^{0}} \\
& -\left\{a_{k}^{(4)}(1 ; x) \frac{d}{d x}\left[\delta a_{k}^{(3)}(1 ; x)\right]+(\delta \ell) \frac{d a_{k}^{(3)}(1 ; x)}{d x} \frac{d}{d x}\left[a_{k}^{(4)}(1 ; x)\right]\right\}_{x=\ell} \\
& -\left\{\delta a_{k}^{(3)}(1 ; x) \frac{d}{d x}\left[a_{k}^{(4)}(1 ; x)\right]\right\}_{x=0}-\left\{\delta a_{k}^{(3)}(2 ; x) \frac{d}{d x}\left[a_{k}^{(4)}(2 ; x)\right]\right\}_{x=0} \\
& -\left\{a_{k}^{(4)}(2 ; x) \frac{d}{d x}\left[\delta a_{k}^{(3)}(2 ; x)\right]+(\delta \ell) \frac{d a_{k}^{(3)}(2 ; x)}{d x} \frac{d}{d x}\left[a_{k}^{(4)}(2 ; x)\right]\right\}_{x=\ell} .
\end{aligned}
$$

4. The left side of Equation (215) is required to represent the indirect-effect term defined in Equation (206) and the unknown values of the functions are eliminated from the right side of Equation (215) by imposing the following relations:

$$
\begin{gathered}
\left\{\frac{d^{2}\left[a_{k}^{(4)}(1 ; x)\right]}{d x^{2}}\right\}_{\alpha^{0}}=2\left\{k_{0} \delta\left(x-x_{r}\right)\right\}_{\alpha^{0}}, 0<x<\ell^{0} ; \\
\frac{d}{d x}\left[a_{k}^{(4)}(1 ; x)\right]=0, \text { at } x=0 ; \\
\left\{\frac{a_{k}^{(4)}(1 ; x)=0, \text { at } x=\ell^{0} ;}{d x^{2}\left[a_{k}^{(4)}(2 ; x)\right]}\right\}_{\alpha^{0}}=4\left\{\delta\left(x-x_{r}\right)\right\}_{\alpha^{0}}, 0<x<\ell^{0} ; \\
\frac{d}{d x}\left[a_{k}^{(4)}(2 ; x)\right]=0, \text { at } x=0 ; \\
a_{k}^{(4)}(2 ; x)=0, \text { at } x=\ell^{0} .
\end{gathered}
$$

5. The relations indicated in Equations (216)-(221) constitute the 4th-LASS to be satisfied by the 4 th-level adjoint function $\mathbf{A}_{k}^{(4)}(2 ; x) \triangleq\left[a_{k}^{(4)}(1 ; x), a_{k}^{(4)}(2 ; x)\right]^{\dagger}$. Inserting the 
equations underlying this 4th-LASS into Equation (206) reduces the latter to the following form:

$$
\begin{aligned}
& \left\{\delta\left[\partial^{3} R / \partial \beta \partial k_{0} \partial Q\right]\right\}_{i n d}=\left\{\int_{0}^{\ell} a_{k}^{(4)}(1 ; x) \frac{d^{2}\left[\delta a_{k}^{(3)}(1 ; x)\right]}{d x^{2}} d x\right\}_{\alpha^{0}} \\
& +\left\{\int_{0}^{\ell} a_{k}^{(4)}(2 ; x) \frac{d^{2}\left[\delta a_{k}^{(3)}(2 ; x)\right]}{d x^{2}} d x\right\}_{\alpha^{0}} \\
& -(\delta \ell)\left\{\frac{d a_{k}^{(3)}(1 ; x)}{d x} \frac{d}{d x}\left[a_{k}^{(4)}(1 ; x)\right]+\frac{d a_{k}^{(3)}(2 ; x)}{d x} \frac{d}{d x}\left[a_{k}^{(4)}(2 ; x)\right]\right\}_{x=\ell} .
\end{aligned}
$$

6. The two variational terms, which appear in the first two terms on the right-side of Equation (222), are now replaced by their equivalent expressions provided on left sides of Equations (207) and (210), respectively, to obtain the following relation:

$$
\begin{aligned}
& \left\{\delta\left[\partial^{3} R / \partial \beta \partial k_{0} \partial Q\right]\right\}_{i n d}=-\left(\delta k_{0}\right)\left\{\frac{1}{k_{0}^{2}} \int_{0}^{\ell} a_{k}^{(4)}(1 ; x)\right\}_{\alpha^{0}} \\
& -(\delta \ell)\left\{\frac{d a_{k}^{(3)}(1 ; x)}{d x} \frac{d}{d x}\left[a_{k}^{(4)}(1 ; x)\right]+\frac{d a_{k}^{(3)}(2 ; x)}{d x} \frac{d}{d x}\left[a_{k}^{(4)}(2 ; x)\right]\right\}_{x=\ell} .
\end{aligned}
$$

Now inserting into Equation (204) the expression of the indirect-effect term obtained in Equation (223), together with the expression of the direct-effect term obtained in Equation (205), yields the following expression for the G-variation $\left\{\delta\left[\partial^{2} R / \partial k_{0} \partial Q\right]\right\}_{\alpha^{0}}$ :

$$
\begin{aligned}
& \left\{\delta\left[\frac{\partial^{3} R}{\partial \beta \partial k_{0} \partial Q}\right]\right\}_{\alpha^{0}} \triangleq\left\{\frac{\partial^{4} R}{\partial \beta \partial k_{0} \partial^{2} Q}\right\}_{\alpha^{0}} \delta Q+\left\{\frac{\partial^{4} R}{\partial \beta \partial^{2} k_{0} \partial Q}\right\}_{\alpha^{0}} \delta k_{0}+\left\{\frac{\partial^{4} R}{\partial^{2} \beta \partial k_{0} \partial Q}\right\}_{\alpha^{0}} \delta \beta \\
& +\left\{\frac{\partial^{4} R}{\partial x_{r} \partial \beta \partial k_{0} \partial Q}\right\}_{\alpha^{0}} \delta x_{r}+\left\{\frac{\partial^{4} R}{\partial \ell \partial \partial k_{0} \partial Q}\right\}_{\alpha^{0}} \delta \ell,
\end{aligned}
$$

where the respective 4 th-order response sensitivities have the following expressions:

$$
\begin{gathered}
\frac{\partial^{4} R}{\partial \beta \partial k_{0} \partial^{2} Q} \equiv 0, \\
\frac{\partial^{4} R}{\partial \beta \partial^{2} k_{0} \partial Q}=2 \int_{0}^{\ell} a_{k}^{(3)}(1 ; x) \delta\left(x-x_{r}\right) d x-\frac{1}{k_{0}^{2}} \int_{0}^{\ell} a_{k}^{(4)}(1 ; x), \\
\frac{\partial^{4} R}{\partial^{2} \beta \partial k_{0} \partial Q} \equiv 0, \\
\frac{\partial^{4} R}{\partial x_{r} \partial \beta \partial k_{0} \partial Q}=-\int_{0}^{\ell}\left[2 k_{0} a_{k}^{(3)}(1 ; x)+4 a_{k}^{(3)}(2 ; x)\right] \delta^{\prime}\left(x-x_{r}\right) d x, \\
\frac{\partial^{4} R \quad}{\partial \ell \partial \beta \partial k_{0} \partial Q}=-\left\{\frac{d a_{k}^{(3)}(1 ; x)}{d x} \frac{d}{d x}\left[a_{k}^{(4)}(1 ; x)\right]+\frac{d a_{k}^{(3)}(2 ; x)}{d x} \frac{d}{d x}\left[a_{k}^{(4)}(2 ; x)\right]\right\}_{x=\ell} .
\end{gathered}
$$

Solving the 4th-LASS analytically yields the following expressions for the components of the 4th-level adjoint function $\mathbf{A}_{k}^{(4)}(2 ; x) \triangleq\left[a_{k}^{(4)}(1 ; x), a_{k}^{(4)}(2 ; x)\right]^{\dagger}$ :

$$
\begin{aligned}
& a_{k}^{(4)}(1 ; x)=\left\{2 k_{0}\left[\left(x-x_{r}\right) H\left(x-x_{r}\right)-\ell+x_{r}\right]\right\}_{\alpha^{0}}, \\
& a_{k}^{(4)}(2 ; x)=\left\{4\left[\left(x-x_{r}\right) H\left(x-x_{r}\right)-\ell+x_{r}\right]\right\}_{\alpha^{0}} .
\end{aligned}
$$


Using the expressions obtained in Equations (230) and (231) together with the expressions for the various functions that have been already obtained in Equations (181)-(184) yields the following closed-form analytical expressions for the 3rd-order sensitivities shown in Equations (226), (228) and (229), respectively:

$$
\begin{gathered}
\frac{\partial^{4} R}{\partial \beta \partial^{2} k_{0} \partial Q}=0, \\
\frac{\partial^{4} R}{\partial x_{r} \partial \beta \partial k_{0} \partial Q}=-2 x_{r}, \\
\frac{\partial^{4} R}{\partial \ell \partial \beta \partial k_{0} \partial Q}=2 \ell .
\end{gathered}
$$

\section{Discussion}

This work has illustrated the application of the "fourth-order comprehensive sensitivity analysis methodology for nonlinear systems (abbreviated as "4th-CASAM-N") to a paradigm model of nonlinear heat conduction. This model was chosen because it admits closed-form expressions for the response sensitivities of all orders, while the algebraic operations involved are sufficiently simple to avoid obscuring the principles underlying the 4th-CASAM-N. The exact explicit expressions of representative response sensitivities of 1st-, 2nd-, 3rd- and 4th-order were determined to illustrate that the 4th-CASAM-N methodology provides sensitivities of linear and nonlinear responses with respect to all model parameters, including correlations, boundary conditions and imprecisely known material boundaries (as would be caused by manufacturing tolerances). The responses chosen for this paradigm model include not only physically measurable quantities but also a synthetic response designed to illustrate the enormous possible reduction in the number of computations when using the 4th-CASAM-N-rather than other methods-for computing response sensitivities.

This illustrative paradigm model has also demonstrated that the implementation of the 4th-CASAM-N requires very little additional effort beyond the construction of the 1st-level adjoint sensitivity system (1st-LASS) for computing the first-order sensitivities. This is because the operators and boundary conditions involved in the 2nd-, 3rd- and 4th-level adjoint sensitivity systems (2nd-LASS, 3rd-LASS and 4th-LASS) are similar to the operators and boundary conditions involved in the 1st-LASS. The largest differences between the 1st-LASS, 2nd-LASS, 3rd-LASS and 4th-LASS arise from the various source terms involved in the adjoint systems of various levels.

Ongoing work aims at generalizing the 4th-CASAM-N methodology to fifth-order and exploring the possibility of developing a comprehensive adjoint sensitivity analysis methodology for nonlinear systems to enable the computation of sensitivities of arbitrarily high order, as a companion methodology to the recently developed "nth-Order Comprehensive Adjoint Sensitivity Analysis Methodology for Response-Coupled Forward/Adjoint Linear Systems (nth-CASAM-L)" [4].

Funding: This research received no external funding.

Data Availability Statement: Not applicable.

Conflicts of Interest: The author declares no conflict of interest.

\section{References}

1. Cacuci, D.G. Fourth-Order Comprehensive Adjoint Sensitivity Analysis Methodology for Nonlinear Systems (4th-CASAM-N): I. Mathematical Framework. Energies 2021, 14, 3335. [CrossRef]

2. Cacuci, D.G. Second-Order Adjoint Sensitivity Analysis Methodology (2nd-ASAM) for Large-Scale Nonlinear Systems: II. Illustrative Application to a Paradigm Nonlinear Heat Conduction Benchmark. Nucl. Sci. Eng. 2016, 184, 31-52. [CrossRef]

3. Cacuci, D.G. The Second-Order Adjoint Sensitivity Analysis Methodology; Taylor \& Francis/CRC Press: Boca Raton, FL, USA, 2018 ; p. 305.

4. Cacuci, D.G. The nth-Order Comprehensive Adjoint Sensitivity Analysis Methodology for Response-Coupled Forward/Adjoint Linear Systems (nth-CASAM-L): I. Mathematical Framework. Energies 2021, 14, 8314. [CrossRef] 